\title{
On the use of watermark-based schemes to detect cyber-physical attacks
}

\author{
Jose Rubio-Hernan ${ }^{1}$, Luca De Cicco ${ }^{2}$ and Joaquin Garcia-Alfaro ${ }^{1 *}$
}

\begin{abstract}
We address security issues in cyber-physical systems (CPSs). We focus on the detection of attacks against cyber-physical systems. Attacks against these systems shall be handled both in terms of safety and security. Networked-control technologies imposed by industrial standards already cover the safety dimension. However, from a security standpoint, using only cyber information to analyze the security of a cyber-physical system is not enough, since the physical malicious actions that can threaten the correct behavior of the systems are ignored. For this reason, the systems have to be protected from threats to their cyber and physical layers. Some authors have handled replay and integrity attacks using, for example, physical attestation to validate the cyber process and to detect the attacks, or watermark-based detectors which uses also physical parameters to ensure the cyber layers.

We reexamine the effectiveness of a stationary watermark-based detector. We show that this approach only detects adversaries that do not attempt to get any knowledge about the system dynamics. We analyze the detection ratio of the original design under the presence of new adversaries that are able to infer the system dynamics and are able to evade the detector with high frequency. We propose a new detection scheme which employs several non-stationary watermarks. We validate the detection efficiency of the new strategy via numeric simulations and via running experiments on a laboratory testbed. Results show that the proposed strategy is able to detect adversaries using non-parametric methods, but it is not equally effective against adversaries using parametric identification methods.
\end{abstract}

Keywords: Cyber-physical security, Control theory, Network security, Networked-control system, Critical infrastructures, Attack detection, Adversary model, Cyber-physical adversary, Attack mitigation

\section{Introduction}

In an effort to reduce complexity and costs, traditional industrial control systems are being upgraded with novel computing, communication, and interconnection capabilities. Industrial control systems that close the loop through a communication network are hereinafter referred to as cyber-physical systems. The adoption of new communication capabilities comes at the cost of introducing new security threats that are required to be holistically handled, both in terms of safety and security (in the traditional ICT sense). The recently coined cyber-physical security term refers to the mechanisms that address this specific challenge [1].

The use of inadequate cyber-physical security mechanisms may have an adverse effect on a vast number of

\footnotetext{
*Correspondence: joaquin.garcia_alfaro@telecom-sudparis.com 'SAMOVAR, Telecom SudParis, CNRS, Université Paris-Saclay, 9 Rue Charles Fourier, 91000, Evry, France
}

Full list of author information is available at the end of the article resources, including assets of private companies, government networks, and mission critical infrastructures [2]. The associated costs, especially in terms of loss of business opportunities and the expenses for fixing the incidents, are expected to be reduced. As a consequence, the issue of the assessment of cyber-physical security mechanisms is a hot research topic.

In this paper, we address security in industrial control systems. Our focus is centered on integrity issues due to the interconnection between cyber and physical control domains in networked-control systems. More specifically, we focus on the adaptation of physical-layer failure detection mechanisms (e.g., systems for the detection of faults and accidents) to handle, as well as, attacks (e.g., replay and integrity attacks conducted by malicious adversaries). We extend the work proposed in [3] which reexamines the security of a specific scheme proposed by Mo et al. in $[4,5]$.

\section{Springer Open}

(c) The Author(s). 2017 Open Access This article is distributed under the terms of the Creative Commons Attribution 4.0 International License (http://creativecommons.org/licenses/by/4.0/), which permits unrestricted use, distribution, and reproduction in any medium, provided you give appropriate credit to the original author(s) and the source, provide a link to the Creative Commons license, and indicate if changes were made. 
The Mo et al. scheme relies on the adaptation of a real-time failure detector based on a linear time-invariant model of the system. Built upon Kalman filters and linearquadratic regulators, the scheme produces authentication watermarks to protect the integrity of physical measurements communicated over the cyber and physical control domains of a networked-control system. Without the protection of the messages, malicious actions can be conducted to mislead the system toward unauthorized or improper actions and affect the availability of the system services.

We show that the Mo et al. detection scheme only works against some integrity attacks. We present two adversary models that can evade the Mo et al. detector. These adversaries are classified based on the algorithm used to obtain the knowledge of the system dynamics in order to carry out the attack (non-parametric [3] and parametric adversaries [6]).

Contributions The main contributions of this paper are summarized as follows:

- We reexamine the effectiveness of the attack detector proposed in $[4,5]$ under new adversary models.

- We show detection weaknesses in $[4,5]$ under the new adversary models.

- Enhanced detector approaches against the new adversaries are presented and validated via numerical simulations and experiments carried out by using a real testbed.

Paper organization Section 2 provides the necessary background for the paper. Section 3 reviews the detector scheme in $[4,5]$, and defines our adversary models and reexamines the security of the detector under the new adversary models. Section 4 adapts the detection scheme in $[4,5]$ to handle the uncovered limitations and validates the resulting approach via numerical simulations. Section 5 presents experimental results based on a laboratory testbed. Section 6 discusses the results. Section 7 reviews some related work. Section 8 concludes the paper.

\section{Background}

\subsection{Industrial control systems}

We assume Industrial Control Systems built upon Supervisory Control and Data Acquisition (SCADA) technologies and Industrial Control Protocols. Such combinations are hereinafter denoted as networked-control systems. Some more information about these systems and protocols follows.

\subsubsection{SCADA}

General term that encompasses well-defined types of field devices, such as: (1) master terminal units (MTUs) and Human Machine Interfaces (HMIs), located at the topmost layer and managing all communications; (2) remote terminal units (RTUs), and programmable logic controllers (PLCs), controlling and acquiring data from remote equipment and connecting with the master stations; (3) sensors and actuators.

The MTUs of a SCADA system are located at the control center of the organization. The MTUs give access to the management of communications, collection of data (generated by several RTUs), data storage, and control of sensors and actuators connected to RTUs. The interface to the administrators is provided via the HMIs.

RTUs are stand-alone data acquisition and control units. They are generally microprocessor-based devices that monitor and control the industrial equipment at the remote site. Their tasks are twofold: (1) to control and acquire data from process equipment (at the remote sites), and (2) to communicate the collected data to a master (supervision) station. Modern RTUs may also communicate between them (either via wired or wireless networks).

PLCs are small industrial microprocessor-based computers. Most significant differences with respect to an RTU are in size and capability. An RTU has more inputs and outputs than a PLC, and much more local processing power (e.g., to postprocess the collected data before generating alerts toward the MTU via the HMI). In contrast, PLCs are often represented by pervasive sensors with communication capabilities. PLCs have two main advantages over traditional RTUs: (1) they are general-purpose devices enforcing a large variety of functions, and (2) they are physically compact.

Sensors are monitoring devices responsible for retrieving measurement related to specific physical phenomena and feed them to the controller. Sensors typically convert a measured quantity to an electrical signal, which is later converted and stored as data. Sensors can be seen as the input function of a SCADA system. The data produced by sensors are sent to the upper layers via the RTUs and the PLCs. Finally, actuators are control devices, in charge of managing some external devices. Actuators translate control signals to actions that are needed to correct the dynamics of the system, via the RTUs and the PLCs.

\subsubsection{Industrial control protocols}

Protocols for industrial control systems must cover regulation rules such as delays and faults [7]. However, few protocols imposed by industrial standards provide security features in the traditional ICT security sense (e.g., confidentiality, integrity, etc.). Details about such ICT security capabilities of representative protocols follows.

Modbus Created in the 70s by Modicon, an American company created in 1968 and absorbed by Schneider Electric. Nowadays, it is one of the most spread protocols, 
probably due to its simplicity and its free license. The Modbus protocol was initially conceived for serial communications. Since 1999, it has been adapted to work over TCP/IP as well. The use of Modbus over TCP/IP allows using SCADA components in heterogeneous environments (i.e., working over IP or serial networks). Moreover, it is possible to use gateways to convert Modbus/TCP to serial Modbus.

From a security standpoint, Modbus does not integrate traditional ICT protection features. For instance, in terms of availability, Modbus/TCP may use some function codes (e.g., ECO4: Server Failure, EC06: Server Busy) as the response of a query from an unavailability device. This way, a controller can point out to availability issues in the absence of responses from one or several devices, or if their responses are error codes. Error in handling is performed at the application layer. The availability of a given equipment is also related to the implementation of the layers below Modbus (e.g., TCP/IP layers) and the nature of the media shared for the exchange of data.

The integrity of a Modbus message is validated by using the TCP layer for Modbus/TCP or by adding a control field (cyclic redundancy check or CRC) for Modbus/Serial. Nevertheless, without authentication of the message, malicious actions can modify the message and recalculate valid CRCs. This kind of validation must be seen only as a protection against transmission errors. Malicious modification of registers, e.g., time windows, is complex but possible. Replay attacks and, in general, integrity attacks, are also possible.

Finally, the Modbus protocol does not implement encryption of messages. Nevertheless, it is possible to implement encryption by encapsulating Modbus/TCP messages under TLS or IPsec tunnels. Confidentiality is not considered as a crucial property in industrial environments. The deployment of encryption solutions can be seen as detrimental given their complexity (e.g., public key infrastructures, manual deployment of keys, etc.), since it may induce to unnecessary latency delays.

PROFINET Suite of industrial protocols operating at different network layers, mainly used in Siemens products. For instance, PROFINET IO is an Ethernet-based protocol associated to the PROFINET suite. Implemented over TCP/IP layers, it allows real-time communication and self-configuration. All equipment implementing PROFINET IO must be certified by the PROFIBUS organization. This certification monitors compliance of software, data models, and integrity in a PROFINET environment.

In 1999, the first security extension of PROFINET was released. Referred to as PROFIsafe, it leverages from PROFINET IO, acting as one of its upper layers. This allows its deployment over less secure networks maintaining acceptable error rate such as IEEE 802.11 and IEEE 802.15.1 standards, while ensuring high availability and backwards compatibility for legacy equipment. Legacy operations can yet use the standard layer, called Black channel, while other operations requiring safety properties, can use the new layers. Those new layers include elements such as continuity control, acknowledgment timeouts, peer authentication, integrity check CRCs, etc.

Åkerberg ans Björkman uncovered in [8] some flaws in the protocol routines associated with the generation of CRCs. Indeed, PROFIsafe meet standards where intentional attacks are not considered a risk. The protocol does not integrate cryptographic features. It only considers protection to cover from unintentional faults. It should not be considered a protection layer against cyber attacks.

Indeed, the PROFINET Safety Guide [9] indicates the use of VPNs whenever ICT security is required. It is important to emphasize that PROFIsafe has been designed to ensure safety and malfunction (e.g., transmission errors). However, it does not ensure security against intentional malicious acts.

DNP3 Short for Distributed Network Protocol version 3, DNP3 is a modern SCADA protocol that includes security extensions, often referred to as DNP3-SA (DNP3 Secure Authentication). DNP3-SA adds features to DNP3 such as protection against replay attacks by ensuring message integrity and authentication. The new features are implemented as new function codes of the original DNP3 protocol. In other words, they are defined at upper layers of the original DNP3 protocol suite, without modifying previous function codes, so that all legacy monitoring and diagnostic tools for DNP3 are still valid. This way, DNP3$\mathrm{SA}$ is compatible with legacy devices that do not require from security support.

The DNP3-SA extensions are expected to be highly scalable. They shall allow changing the security algorithms, keys sizes, and other parameters to meet future conditions of state-of-the-art installations. Nevertheless, both DNP3 and DNP3-SA are relatively complex protocols. The DNP3-SA extensions are relatively young, and the first DNP3-SA products may present vulnerabilities. They do not provide protection against confidentiality attacks either. For the time being, DNP3-SA has not been largely deployed. Given the pace of industrial systems (whose upgrades are often superior to decades), it may take quite long before DNP3-SA is fully tested over large environments.

Ethernet/IP Nowadays maintained by ODVA Inc. (formerly Open DeviceNet Vendors Association, Inc.), Ethernet/IP is an industrial protocol that relies on the use 
of CIP (Common Industrial Protocol) over standard Ethernet frames [10]. According to [11], Ethernet/IP was the most widely deployed protocol on industrial environments in 2009, with about $30 \%$ of active nodes (about 5 million nodes).

Communications over Ethernet/IP can either be in an unconnected mode, using TCP/IP in a client/server model, or they can be in connected mode. In that case, resources are reserved to create a link between two users; UDP/IP and multicast transmissions are employed to make latency as small as possible to enforce real-time constraints. In fact, Ethernet/IP inherits from Ethernet all its security issues. Moreover, Ethernet/IP environments mainly use UDP/IP connections, and rarely use security mechanisms (in a traditional ICT sense), due to performance and real-time constraints [12]. CIP does not implement security features either. Thus, we can consider some concerns such as message hijacking, disclosure of communication and configuration details, injection of unauthorized frames, etc.

\subsubsection{Networked-control systems (NCSs)}

NCSs are spatially distributed systems whose control loops are connected through communication networks. The communication network connects the different components of a traditional control system, i.e., the controller, sensors, and actuators. Examples include smart grids, smart vehicles, and water distribution systems. The use of a communication network to connect the different components of a control system adds more flexibility in the system and reduces the implementation cost of new installations. However, the use of a communication network to decentralize traditional control systems comes at the price of an increased control design complexity. For instance, the analysis and design of the overall system has also to deal with new theoretical challenges due to, for instance, loss of measurements and time-varying sampling [13]. The integration of the control system (often referred as physical-space) with the communication network (cyber-space) creates a new degree of interaction between these two domains [14].

Communication protocols used in traditional control systems are required to comply with the constrains imposed by industrial standards (e.g., to cover regulation roles such as delay and faults). Indeed, prominent industrial control protocols (e.g., Modbus, PROFINET, and Ethernet/IP) are not designed to provide security from a traditional information or network perspective. However, current NCSs use these protocols over TCP/IP or UDP/IP communications (e.g., Modbus over TCP, PROFINET over TCP, and Ethernet/IP over TCP or UDP). Although such combinations can provide some security elements at either their transport or network layers, this is not enough to ensure control-data protection. At the same time, traditional control systems come with already existing mechanisms to handle failures. Such mechanisms are expected to detect faults and avoid accidents. Nevertheless, classical mechanisms conceived to detect failures in control systems are not able to detect intentional actions from malicious adversaries holding enough knowledge about the systems. Cryptography can be used to ensure the integrity of control-data. This may partially solve the aforementioned problem. Without underestimating cryptographic solutions, in this paper, we consider a complementary solution proposed by Mo et al. $[4,5]$ to protect the integrity of the physical sensor measurements by means of authentication watermarks. Such watermarks are enforced by adapting a real-time failure detector based on linear time invariant models of industrial control systems. This security solution allows to improve system security in cases where, e.g., cyber-adversaries bypass security measures at upper protocol layers. We present some representative examples in the following section.

\subsection{Cyber-physical attacks}

The use of communication networks and IT components in traditional control systems paves the way to new vulnerability issues. The attacks against these setups are named cyber-physical attacks. These threats may target physical processes through the network. In [15], Texeira et al. propose a taxonomy of cyber-physical attacks based on the resources of the adversaries. Such resources are mainly measured in terms of adversary knowledge (e.g., a priori knowledge of the adversary about the system and its security measures). Indeed, the knowledge of the adversary about the system is the main resource in order to build up complex attacks, as well as to make them undetectable. Based on the degree of the adversary knowledge, the attacks may succeed at violating system properties, such as availability and integrity, as well as to obtaining operational information about the system to make the attacks undetectable.

Figure 1 shows an adversary conducting a cyberphysical attack. The $\bigoplus$ symbol in the figure represents a summing junction, i.e., a linear element that outputs the sum of a number of input signals. In a nutshell, the adversary succeeds at modifying some plant measurements, by recording and replicating previous measurements corresponding to normal operation conditions. Then, the adversary modifies the control input $u$ to affect the system state and disrupt normal operation conditions. If, on the one hand, the adversary is not required to have the knowledge of the system process model, on the other hand, access to all sensors (i.e., it has access to all components of the vector $y$ ) or insecure communication protocols is required to carry out a successful attack. This type of adversary is undetectable with a monitor detector which only verifies faulty measurements. 


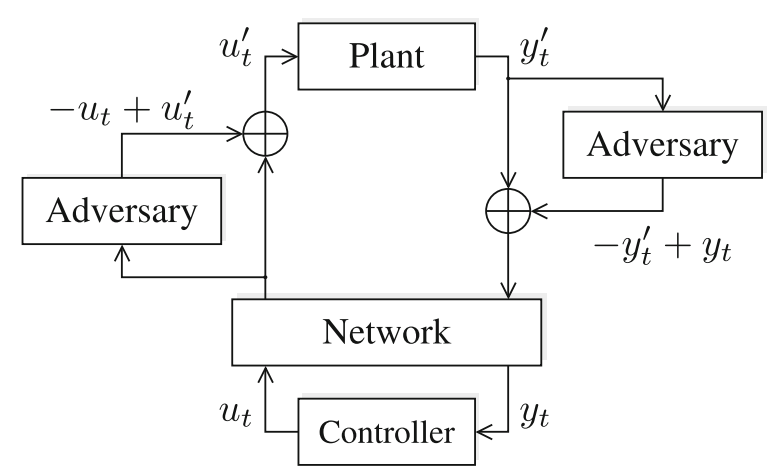

Fig. 1 Sample representation of a cyber-physical attack against a networked-control system

Adversaries conducting more powerful injection attacks are expected to modify some of the plant measurements, either by targeting the individual sensors or their communication channels in case of insecure communication protocols. Sophisticated variation of the attack in Fig. 1 includes (1) bias-injection cyber-physical attacks, in which the new data injected by the adversary corresponds to a bias from the legitimate data, with the aim of leading the system to wrong control decisions (e.g., to cause malfunction in the long-term); and (2) geometricinjection cyber-physical attacks, in which the bias is gradually injected. The attack may remain undetected when data compatible with the system dynamics are injected, potentially leading the system to irreversible damages [15]. Another undetectable attack is the covert attack, where the adversary knows perfectly the system model. This attack is defined in [16], and the author concludes that it is not possible to be detected.

Techniques to prevent the aforementioned cyberphysical attacks exist. In [17], Arvani et al. describe a signal-based detector method, using discrete wavelet transformations. Do et al. study in [18] strategies for handling cyber-physical attacks using statistical detection methods. Mo et al. propose in $[4,5]$ the use of watermark-based detection by adapting traditional failure detection mechanisms (e.g., detectors to handle faults and errors). In the following sections, we elaborate further on the watermark-based technique by Mo et al. discuss about some security limitations and propose an improved technique.

\section{Watermark-based attack detection}

In $[4,5]$, a watermark-based strategy is proposed to detect replay and injection attacks against cyber-physical systems. This section reviews the mechanism proposed in $[4,5]$ and assesses its performance when a new adversary model, that we name cyber-physical adversary, is employed. In particular, this section is organized as follows: in Section 3.1 we provide some necessary definitions and background concerning the class of control systems considered in this paper; Section 3.2 describes the attack detection scheme proposed in $[4,5]$; in Section 3.3, we propose the cyber-physical adversaries; finally, in Section 3.4 we show methods that can be employed by the adversary to mislead the watermark-based detector. The notation used hereinafter is summarized in Table 1.

\subsection{Definitions and background}

We consider plants of industrial control systems that can be mathematically modeled as discrete linear timeinvariant (LTI) systems. It is worth mentioning that a mathematical model provides a rigorous way to describe the dynamical behavior of a given system. In the following, we denote with $X(z)$ the $Z$-transform of a signal $x_{t}$, where $t$ is the time-discrete variable. Such class of systems can be described as follows:

$$
z X(z)=A X(z)+B U(z)+W(z)
$$

where $X(z)$ is the $Z$-transform of $x_{t} \in \mathbb{R}^{n}$, the vector of the state variables (or state) at the timestep $t, U(z)=Z\left\{u_{t}\right\}$ is the $Z$-transform of $u_{t} \in \mathbb{R}^{p}$, the control signal, and $W(z)$ is the $Z$-transform of $w_{t} \in \mathbb{R}^{n}$, the process noise that is assumed to be a white noise with zero mean and variance $Q$, i.e., $w_{t} \sim N(0, Q)$. Moreover, $A \in \mathbb{R}^{n \times n}$ and $B \in \mathbb{R}^{n \times p}$ are, respectively, the state matrix and the input matrix.

A static relation maps the state $X(z)$ to the system output $Y(z)$, the $Z$-transform of $y_{t} \in \mathbb{R}^{m}$ :

$$
\begin{aligned}
Y(z) & =C X(z)+V(z) \\
& =C(z I-A)^{-1}(B U(z)+W(z))+V(z)
\end{aligned}
$$

where $C \in \mathbb{R}^{m \times n}$ is the output matrix. The value of the output vector $Y(z)$ represents the measurement produced by the sensors that is affected by a noise $V(z)$ that is the $Z$ transform of $v_{t}$ assumed as a white noise with zero mean and variance $R$, i.e., $v_{t} \sim N(0, R)$.

For the class of systems defined above, a widely used and effective control technique is the linear quadratic Gaussian (LQG) approach. The overall goal of an LQG controller is to produce a control law $U(z)$ such that a quadratic cost $J$, that is function of both the state $x_{t}$ and the control input $u_{t}$, is minimized:

$$
J=\lim _{n \rightarrow \infty} E\left[\frac{1}{n} \sum_{j=0}^{n-1}\left(x_{j}^{T} \Gamma x_{j}+u_{j}^{T} \Omega u_{j}\right)\right]
$$

where $\Gamma$ and $\Omega$ are positive definite cost matrices [19]. 
Table 1 Notations used in this paper

\begin{tabular}{|c|c|c|}
\hline$A$ & : & State matrix. \\
\hline B & : & Input matrix. \\
\hline C & : & Output matrix. \\
\hline$W(z)$ & : & Process noise. \\
\hline Q & : & Process noise variance. \\
\hline$V(z)$ & : & Output noise. \\
\hline$R$ & : & Output noise variance. \\
\hline$X$ or $X(z)$ & : & Vector of state variables. \\
\hline$U \operatorname{or} U(z)$ & : & Control input vector. \\
\hline$Y$ or $Y(z)$ & : & Vector of the sensors measurements. \\
\hline$U^{*} \operatorname{or} U^{*}(z)$ & : & Optimal control input vector. \\
\hline$\Delta U$ or $\Delta U(z)$ & : & Watermark. \\
\hline$\Delta U(z)^{(i)}$ & : & Multi-watermark. \\
\hline$Y^{\Delta U}(z)$ & : & Output due to the watermark. \\
\hline$Y^{\prime}$ or $Y^{\prime}(z)$ & : & Measurements injected by the adversary. \\
\hline$U^{\prime} \operatorname{or} U^{\prime}(z)$ & : & Control inputs injected by the adversary. \\
\hline$\hat{x}$ or $\hat{X}(z)$ & : & Vector of estimated state variables. \\
\hline$\hat{X}^{(-)}$or $\hat{X}^{(-)}(z)$ & : & $\begin{array}{l}\text { Vector of estimated state variables before applying } \\
\text { the rectification. }\end{array}$ \\
\hline$\hat{X}^{(+)}$or $\hat{X}^{(+)}(z)$ & : & $\begin{array}{l}\text { Vector of estimated state variables after applying } \\
\text { the rectification. }\end{array}$ \\
\hline$K_{f}$ & : & Kalman gain. \\
\hline$p^{(-)}$ & : & A priori error covariance. \\
\hline$p^{(+)}$ & : & A posteriori error covariance. \\
\hline L & & Feedback grain. \\
\hline$S$ & : & Riccati equation solution. \\
\hline J & : & Quadratic cost. \\
\hline$\Delta \mathrm{J}_{S}$ & : & $\begin{array}{l}\text { Increment of quadratic cost due to the } \\
\text { single-watermark. }\end{array}$ \\
\hline$\Delta J_{m}$ & : & $\begin{array}{l}\text { Increment of quadratic cost due to the } \\
\text { multi-watermark. }\end{array}$ \\
\hline$E[\Delta u]$ & : & Offset of $\Delta u$. \\
\hline $\operatorname{Var}[\Delta u]$ & : & Variance of $\Delta u$. \\
\hline $\mathcal{W}$ & : & LMS weight matrix. \\
\hline$D R$ & : & Detection ratio. \\
\hline$g_{t}$ & : & Alarm signal. \\
\hline$\hat{T}$ & : & Samples eavesdropped by the adversary. \\
\hline $\mathcal{P}$ & $:$ & Co-variance of the i.i.d. Gaussian signal. \\
\hline$r(z)$ & : & Residue. \\
\hline$\gamma$ & : & Detection threshold. \\
\hline$\Gamma$ and $\Omega$ & : & Ponderation matrices. \\
\hline$\left(n_{0} \ldots n_{m}\right)$ & : & Weight of the polinomial N(z). \\
\hline$\left(d_{0} \ldots d_{n}\right)$ & : & Weight of the polinomial D(z). \\
\hline FN & : & False negatives. \\
\hline$F P$ & : & False positives. \\
\hline$A D$ & $:$ & Samples detected. \\
\hline$S A$ & $:$ & Samples under attack. \\
\hline
\end{tabular}

It is well-known that such a control problem has, under some unrestrictive technical conditions, an optimal solution that, thanks to the separation principle, is made of two components that can be designed independently:

1. a Kalman filter that, based on the noisy measurements, produces an optimal state estimation $\hat{X}(z)$ of the state $X(z)$;

2. a linear quadratic regulator (LQR) that, based on the state estimation $\hat{X}(z)$, provides the control law $U(z)$ that solves the LQR problem (cf. Eq. (3)).

Let us briefly illustrate how these two components are designed. The Kalman filter estimates the state as follows:

- Predict (a priori) system state $\hat{X}^{(-)}(z)$ and covariance:

$$
\begin{aligned}
\hat{X}^{(-)}(z) & =A \hat{X} z^{-1}+B U z^{-1} \\
P^{(-)} & =A\left(P^{(+)} z^{-1}\right) A^{T}+Q
\end{aligned}
$$

- Update parameters and (a posteriori) system state and covariance:

$$
\begin{aligned}
K_{f} & =\left(P^{(-)} C^{T}\right)\left(C P^{(-)} C^{T}+R\right)^{-1} \\
\hat{X}^{(+)} & =\hat{X}^{(-)}(z)+K_{f}\left(Y(z)-C \hat{X}^{(-)}(z)\right) \\
P^{(+)} & =\left(I-K_{f} C\right) P^{(-)}
\end{aligned}
$$

where $K_{f}$ and $P^{(+)}$denote, respectively, the Kalman gain and the a posteriori error covariance matrix, $I$ is the identity matrix.

The optimal control law $U(z)$ provided by the LQR is a linear controller:

$$
U(z)=L \hat{X}^{(+)}(z)
$$

where $L$ denotes the feedback gain of a linear-quadratic regulator $(L Q R)$ which minimizes the control cost (cf. Eq. 3) and it is defined as follows (cf. $[4,5]$ for further details):

$$
L=-\left(B^{T} S B+\Omega\right)^{-1} B^{T} S A,
$$

with $S$ being the matrix that solves the following discrete time algebraic Riccati equation:

$$
S=A^{T} S A+\Gamma-A^{T} S B\left[B^{T} S B+\Omega\right]^{-1} B^{T} S A .
$$

\subsection{The $\chi^{2}$ detector}

This section briefly describes the detection scheme proposed in $[4,5]$. The procedure is applicable to discrete LTI plants controlled by a LQG controller as detailed in Section 3.1. 
Before presenting the detection scheme, we provide a definition of the adversary model considered in $[4,5]$ :

Definition 3.1 An attacker that has the ability to eavesdrop all the messages containing the sensor outputs $Y(z)$ and to inject messages with an arbitrary signal $Y^{\prime}(z)$ to conduct malicious actions is defined a cyber adversary.

Remark 3.2 Notice that the definition given above does not suppose that the attacker possesses (or makes attempts to gather) any knowledge about the system model.

We denote with $U^{*}(z)$ the output of the LQR controller given by Eq. (4) and with $U(z)$ the control input that is sent to the plant (cf. Eq. (1)). The idea is to superpose to the optimal control law $U^{*}(z)$ a watermark signal, $\Delta U(z)=Z\left\{\Delta u_{t}\right\}, Z$-transform of $\Delta u_{t} \in \mathbb{R}^{p}$ that serves as an authentication signal. Thus, the control input $U(z)$ is given by

$$
U(z)=U^{*}(z)+\Delta U(z)
$$

The watermark signal is a Gaussian random signal with zero mean that is independent both from the state noise $W(z)$ and the measurement noise $V(z)$. Such an authentication watermark is expected to detect replay and integrity attacks modeled by the adversary defined above. Now that the optimal control law $U^{*}(z)$ is equipped with the authentication signal $\Delta U(z)$, a detector - physically co-located with the controller - can be designed having the goal of generating alarms when an attack takes place. Toward this end, $[4,5]$ propose to employ a $\chi^{2}$ detector, a well-known category of real-time anomaly detectors classically used for fault detection in control systems [20], for the purpose of attack detection.

Figure 2 shows the overall control system equipped with the attack detector proposed in $[4,5]$.

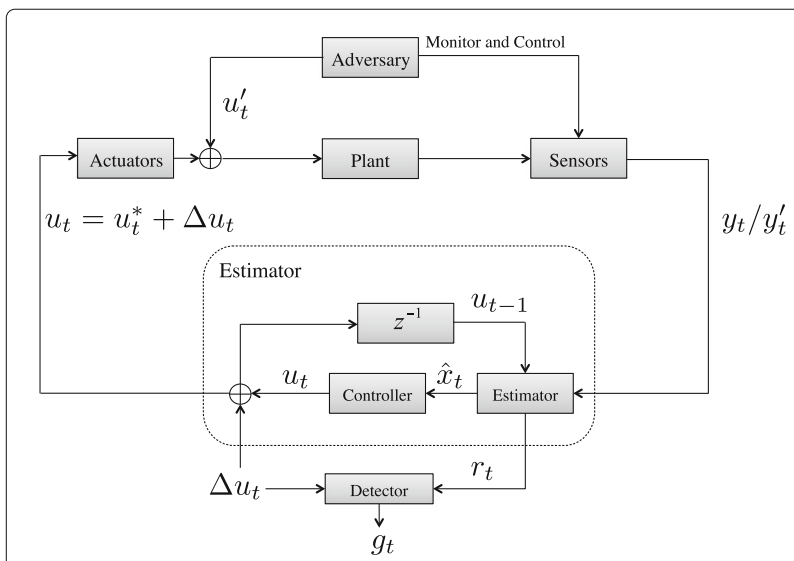

Fig. 2 Overall control system equipped with the watermark-based detection scheme in $[4,5]$
An alarm signal $g_{t}$ is computed based on the residues $r_{t}=Z^{-1}\{r(z)\}$, where $r(z)=Y(z)-C \hat{X}^{(-)}$generated from the estimator. Then, $g_{t}$ is compared with a threshold $\gamma$ to decide whether the system is in a normal state. The threshold is tuned to minimize false alarms $[4,5]$. The alarm signal $g_{t}$ is computed as follows:

$$
g_{t}=\sum_{i=t-w+1}^{t}\left(r_{i}\right)^{T} \mathcal{P}^{-1}\left(r_{i}\right)
$$

where $w$ is the size of the detection window and $\mathcal{P}=$ $\left(\mathrm{CPC}^{T}+R\right)$ is the co-variance of an independent and identically distributed (i.i.d.) Gaussian input signal from the sensors.

The system is considered not under attack if $g_{t}<\gamma$, otherwise if $g_{t} \geq \gamma$ the system is considered to be under attack and the detector generates an alarm.

\subsection{Cyber-physical adversary}

Let us assume the system employs the detector described in Section 3.2, so that the controller superposes its output with an authentication watermark $\Delta U(z)$. At steady-state, i.e., after the transient has been exhausted, the output of the system can be considered as the sum of its steady-state value and a component that is due to watermark signal that shall be only known by the controller.

Let us now introduce an enhanced adversary that is aware of the fact that the system employs the $\chi^{2}$ detector presented above. Since the detector is based on a stationary watermark signal $\Delta U(z)$, we will show that an adversary that is able to extract the model of the system from the control law $U(z)$ and the sensor measurement $Y(z)$ is able to conduct an attack while remaining undetected.

Definition 3.3 An attacker that, in addition to the capabilities of the cyber adversary, is also able to eavesdrop the messages containing the output of the controller $U(z)$ with the intention of improving its knowledge about the system model using a parametric or non-parametric identification model, is defined as a cyber-physical adversary.

Based on the way to model the system's behavior, two different cyber-physical adversaries can be defined.

Definition 3.4 An attacker that only uses the previous input and output of the system to identify the system model is defined as a non-parametric cyber-physical adversary.

Remark 3.5 A non-parametric cyber-physical adversary can use, e.g., a finite impulse response (FIR) model identification tool to identify the system model [21]. In Fig. 2, the signals $u_{t}^{\prime}=Z^{-1}\left\{U^{\prime}\right\}$ and $y_{t}^{\prime}=Z^{-1}\left\{Y^{\prime}\right\}$ are assumed 
to be, respectively, the output of the controller and the output of the measurement when an attack is taking place. We denote with $\Delta u^{\prime}=Z^{-1}\left\{\Delta U^{\prime}\right\}$ the watermark guessed by the non-parametric cyber-physical adversary.

Definition 3.6 An attacker that is able to estimate the parameters of the system using input and output data to mislead the controller detector is defined as a parametric cyber-physical adversary.

Remark 3.7 A parametric cyber-physical adversary is able to estimate the parameters of the system using input and output data to mislead the controller detector. This adversary can use an ARX (autoregressive with exogenous input) model or an ARMAX (autoregressive-moving average with exogenous input) to estimate the model [22].

We assume that the main constraint of this adversary is the energy spent to eavesdrop and analyze the communication data, i.e., the number of samples eavesdropped to obtain the system model parameters.

Proposition 3.8 A cyber-physical adversary that is able to exactly estimate the system controlled by the controller cannot be detected by the $\chi^{2}$ detector (cf. Eq. 6).

Proof Without loss of generality, we assume an attack is started at time $T_{0}$, and we compute the residues $r_{t}$ for $t \in\left[T_{0}, T_{0}+T-1\right]$ :

$$
Z\left\{r_{t}\right\}=Y^{\prime}(z)-C \hat{X}^{(-)} z^{-\left(t-T_{0}-1\right)}
$$

Moreover, it is easy to show that the following holds:

$$
\begin{aligned}
\hat{X}^{(-)} z^{-\left(t-T_{0}-1\right)}= & Z\left\{\hat{x}_{t \mid t-T}^{\prime}+\mathcal{A}^{t-T_{0}}\left(\hat{x}_{T_{0} \mid T_{0}-1}-\hat{x}_{T_{0} \mid T_{0}-1}^{\prime}\right)\right. \\
& \left.+\sum_{i=0}^{t-T_{0}-1}\left(\mathcal{A}^{i} B\left(\Delta u_{t-1-i}-\Delta u_{t-1-i}^{\prime}\right)\right)\right\} \\
= & \hat{X}^{\prime(-)} z^{-(t-T-1)}+\left(\mathcal{A}^{t-T_{0}}\right) \\
& \times\left(\hat{X}^{(-)}-\hat{X}^{(-)}\right) z^{-\left(t-T_{0}-1\right)} \\
& +\sum_{i=0}^{t-T_{0}-1}\left(\mathcal{A}^{i} B(\Delta U(z)\right. \\
& \left.\left.-\Delta U^{\prime}(z)\right)\right) z^{-\left(t-T_{0}-1-i\right)}
\end{aligned}
$$

where $\hat{X}^{\prime}$ is the estimated state when the system is under attack, and $\mathcal{A}=(A+B L)(I-K C)$ is a stable matrix $[4,5]$. Substitution of (8) in (7) yields:

$$
\begin{aligned}
Z\left\{r_{t}\right\} & =\underbrace{Y^{\prime}(z)-C \hat{X}^{\prime(-)}}_{\text {First term }} \\
& -\underbrace{C\left(\mathcal{A}^{t-T_{0}}\right)\left(\hat{X}^{(-)}-\hat{X}^{\prime(-)}\right) z^{-\left(t-T_{0}-1\right)}}_{\text {Second term }} \\
& -\underbrace{C \sum_{i=0}^{t-T_{0}-1}\left(\mathcal{A}^{i} B\left(\Delta U(z)-\Delta U^{\prime}(z)\right) z^{-\left(t-T_{0}-1-i\right)}\right)}_{\text {Third term }}
\end{aligned}
$$

Let us consider separately the three terms in the equation written above: the first term follows the same distribution of $Y^{\prime}(z)-C \hat{X}^{\prime(-)}$; since $\mathcal{A}$ is asymptotically stable - i.e., all its eigenvalues are inside the open unit disk of the complex plane - the second term converges exponentially fast to zero. In fact, the entries of $\mathcal{A}^{t-T_{0}}$ converge exponentially fast to zero. Now, if the third term is equal to zero, the dynamics of $Z\left\{r_{t}\right\}$ would recover the dynamics of the residues when no attack is undergoing and thus, the attack would not be detected. Under the hypothesis of this proposition, the adversary knows exactly the watermark signal and thus $\Delta U(z)=\Delta U^{\prime}(z)$ which makes the third term equal to zero and concludes the proof.

\subsection{Acquiring the watermark signal model}

Motivated by Definition 3.4, we show now a practical method that can be used to acquire the watermark signal $\Delta u_{t}$. In particular, we propose an adversary that employs a well known LMS (least mean square) adaptive FIR filter, a non-parametric identification model, with the purpose of running an online identification of the system model. With the identified model, it is possible to obtain the watermark and, finally, using it to authenticate messages with the aim of driving the system to an undesired state.

We denote with $p$ the LMS filter order and with $\mu$ its step size. The step size $\mu$ is upper bounded by $2 / \lambda_{\max }$, where $\lambda_{\max }$ is the maximum eigenvalue of the autocorrelation matrix $R=E\left[X X^{H}\right]$, where $X^{H}$ is the Hermitian transpose, or conjugate transpose, of $X$. Observe that if $\mu$ is chosen too small, the time to converge to optimal weights tends to be large [23]. The adversary initializes the weight matrix $\mathcal{W}$ to be equal to the zero matrix. Then, the adversary's algorithm shown in Algorithm 1 is run online. It is worth noting that in this algorithm $X[x(t-$ $p+1), \ldots, x(t)]$ is the input signal vector, $e(t)$ is the error vector, $\bar{e}(t)$ is its complex conjugate, and $d(t)$ is the desired output signal. 


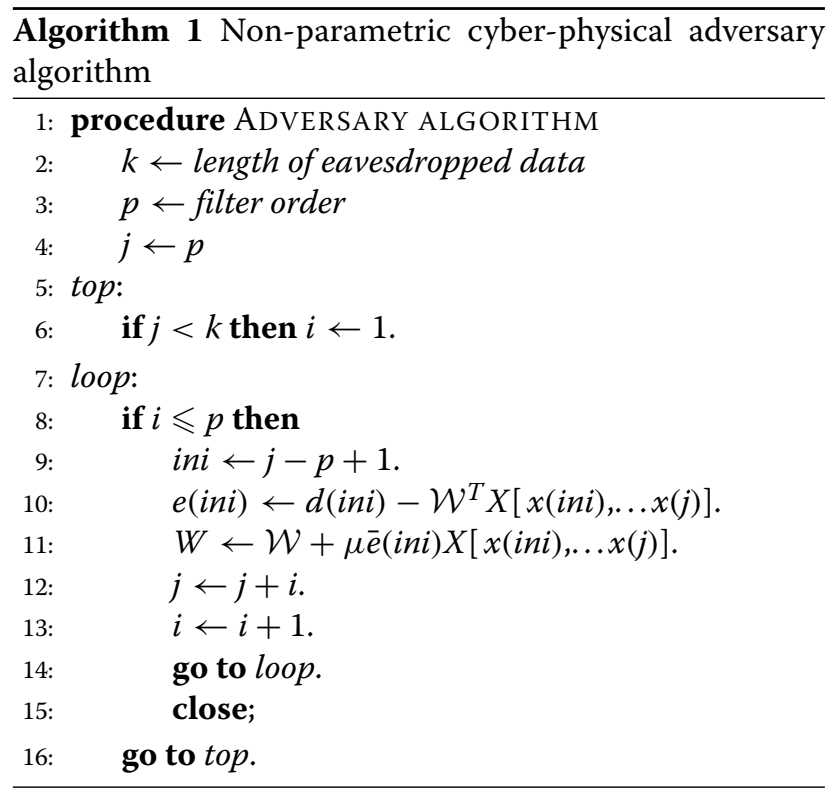

Once the system model has been identified, the adversary is able to extract the watermark and carry out the replay attack. In particular, the adversary follows the steps described below:

1. Eavesdropping of $U(z)$ and $Y(z)$ and decomposition. The adversary captures both the control law $U(z)$ and the sensors output $Y(z)$ to make the decomposition between the information data and the watermark using the LMS filter as a noise cancellation adaptive filter. With this first step, we are able to separate $U^{*}(z)$ and the watermark $\Delta U(z)$ starting from $U(z)$. Notice that, since the system is linear, it follows from the superposition principle that $Y(z)=Y^{*}(z)+Y^{\Delta U}$, being $Y^{*}(z)$ as the output due to $U^{*}(z)$ and $Y^{\Delta U}(z)$ as the output due to the watermark $\Delta U(z)$.

2. Acquiring the weight matrix, $\mathcal{W}$. To acquire the weight matrix $\mathcal{W}$, the adversary uses the LMS adaptive filter described before; as a system identification method.

3. Computing the attack sensor measurement $Y^{\prime}(z)$. The adversary attacks the system by sending fake sensor measurements $Y^{\prime}(z)$, where $Y^{\Delta U}(z)$ is computed using the watermark $\Delta U(z)$ as follows:

$$
\begin{gathered}
Y^{\Delta U}(z)=\mathcal{W}^{T} \Delta U(z) \\
\text { and } Y^{\prime}(z)=Y^{*}(z) z^{-1}+Y^{\Delta U}(z) .
\end{gathered}
$$

In the remainder of this section, we show via numerical simulations that the detection mechanism proposed in $[4,5]$ is not sufficiently robust and is not able to detect cyber-physical adversaries (cf. Section 3.3) that are able to identify the system model by eavesdropping the data channel.
In order to simulate the scenario proposed in this section, we use a simplified version of the Tennesse Eastman control challenge problem [24] (also used in [25]). This system simulates a MIMO system of order $n=7$ with $p=4$ inputs and $m=4$ outputs. In particular, the model of the discrete LTI system described by Eqs. (1)-(2) is defined by the following matrices:

$A=\left[\begin{array}{ccccccc}0.987 & 0 & 0 & 0 & 0 & 0 & 0 \\ 0 & 0.895 & -0.025 & 0 & 0 & 0 & 0 \\ 0 & 0.036 & 0.999 & 0 & 0 & 0 & 0 \\ 0 & 0 & 0 & 1 & -0.008 & 0 & 0 \\ 0 & 0 & 0 & 0.005 & 0.960 & 0 & 0 \\ 0 & 0 & 0 & 0 & 0 & 0.999 & 0 \\ 0 & 0 & 0 & 0 & 0 & 0 & 0.990\end{array}\right]$,

$B=\left[\begin{array}{cccc}0.149 & 0 & 0 & 0 \\ 0 & 0 & 0 & 0.071 \\ 0 & 0 & 0 & 0.001 \\ 0.380 & 0 & -0.096 & 0 \\ 1.000 & 0 & -0.096 & 0 \\ 0 & 0.038 & 0 & 0 \\ 0 & 0 & 0 & 0.075\end{array}\right]$

$C=\left[\begin{array}{ccccccc}0.151 & -0.076 & 0 & 0 & 0 & 0 & 0 \\ 0 & 0 & 0 & 0 & 1 & 0 & 0 \\ 0 & 0 & 0 & 0 & 0 & 0.040 & 0 \\ 0 & 0 & 0 & 0 & 0 & 0 & 0.133\end{array}\right]$

Moreover, the co-variance matrices are equal to $Q=$ $0.01 I$ and $R=I$, whereas the cost matrices are $\Gamma=1.5 I$ and $\Omega=10 I$.

To validate our approach, we compare the system dynamics considering the two adversaries described above. Figure 3a, c shows the plant dynamics and the state estimated by the controller in the case of a cyber adversary. Figure 3a shows that the adversary is able to drive the state components to an undesired value. Nevertheless, the controller, misled by the adversary, does not perceive such situation (cf. Fig. 3c). Figure 3b, d shows the dynamics of the plant and the ones of the controller under a non-parametric cyber-physical adversary model which exhibits the same behavior described above for the case of the cyber adversary.

Let us now contrast the performance of the detector described in Section 3.2 when detecting either the cyber or the non-parametric cyber-physical adversary. Toward this end, Fig. 3e, $\mathrm{f}$ shows the value of the alarm signal $g_{t}$ produced by the same $\chi^{2}$ detector in the case of the cyber adversary (cf. Fig. 3e) and the non-parametric 


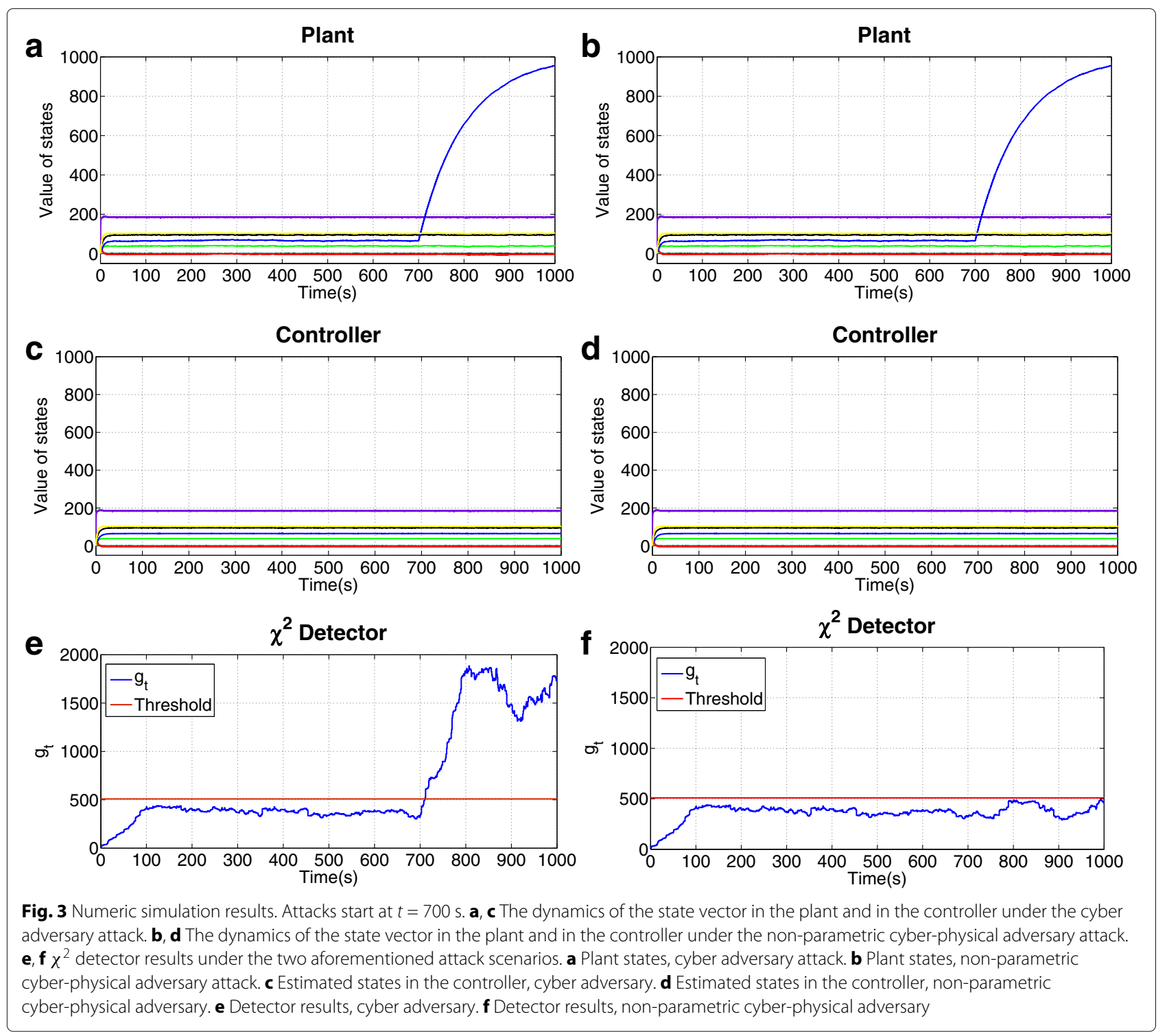

cyber-physical adversary (cf. Fig. 3f). Figure 3e shows that the detector is able to detect the cyber adversary thanks to the added watermark signal as soon as the attack starts at $t=700 \mathrm{~s}$. However, Fig. $3 \mathrm{f}$ shows that the same detector is not able to detect the non-parametric cyberphysical adversary since $g_{t}$ does not exceed the threshold $\gamma$ during the attack. In order to quantify the detector performance, we define a detection ratio (DR) metric as follows:

$$
\mathrm{DR}=\frac{\sum_{t=T_{0}}^{T_{0}+T_{a}} \mathbb{1}_{g_{t} \geq \gamma}}{T_{a}}
$$

where $T_{a}$ is the attack duration, and $\mathbb{1}$ is the indicator function whose output is equal to 1 if the Boolean condition given as its argument $\left(g_{t} \geq \gamma\right)$ is true; or 0 oth- erwise. In a nutshell, $\mathrm{DR} \in[0,1]$ can be considered as an efficiency index for the detector: DR is equal to 1 when the attack is always detected; and it is equal to 0 when the attack is always undetected.

Figure 4 shows the CDF (cumulative distribution function) of the detection ratio obtained by measuring DR for 200 simulations both in the case of the non-parametric cyber-physical and the cyber adversary. The figure shows that the detection scheme proposed in $[4,5]$ is able to provide a median detection ratio that is larger than 0.9 when a cyber adversary attacks the system. However, using a non-parametric cyber-physical adversary that acquires the watermark, the median detection ratio drops to around 0.2 . This quantitatively shows that the detection strategy proposed in $[4,5]$ is not sufficiently robust for security. 


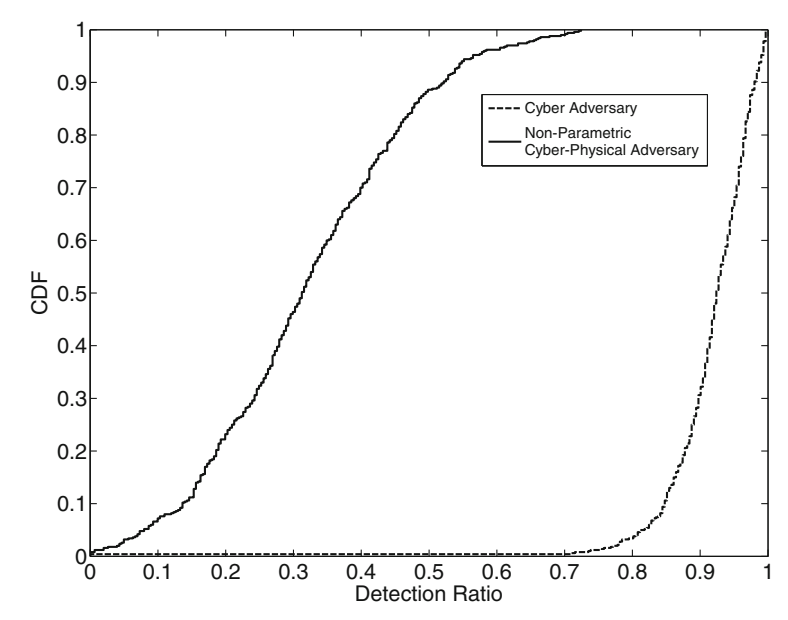

Fig. 4 Cumulative distribution function (CDF) of the detection ratio associated with the $\chi^{2}$ detector (see Section 3.2), obtained by measuring the DR metric (see Eq. 9) for 200 simulations (both cyber and non-parametric cyber-physical adversary cases)

\section{Multi-watermark-based attack detection}

In the previous section, we have defined three different kinds of adversaries who use different vulnerabilities of a control system to carry out attacks; cyber-adversary, non-parametric cyber-physical adversary, and parametric cyber-physical adversary. In this section, we propose a detection scheme that extends the one presented in $[4,5]$, in order to detect non-parametric cyber-physical adversaries. We also study the performance loss of the new detection scheme with regard to the one presented in $[4,5]$.

\subsection{On the use of multi-watermark signals}

The goal of our new detection scheme is to increase the difficulty in retrieving the authentication watermark $\Delta U(z)$ from the control signal $U(z)$, so that the probability of detecting an attack from a non-parametric cyber-physical adversary can be increased. We assume that the control system under attack employs exactly the same type of controller and the same detection strategy presented in Section 3.2. The only difference in the proposed detection scheme is the way that the watermark signal $\Delta U(z)$ is generated. The control input $U(z)$, as in the case of the detection scheme presented in Section 3.2, is computed as the superposition of the optimal control signal $U^{*}(z)$ produced by the LQR controller and a given multi-watermark signal $\Delta U(z)$. The idea is to construct the authentication watermark signal by switching between $N$ different and independent processes with different co-variance and average (offsets). More precisely, the non-stationary watermark $\Delta U$ is obtained by periodically switching, with a period $T$, between $N$ signals $\Delta U^{(i)}$, with $i \in \mathcal{I}=\{0,1, \ldots, N-1\}$, extracted by different stochastic processes. Hence, the watermark signal $\Delta U(z)$ can be formalized as follows:

$$
\Delta U(z)=Z\left\{\Delta u_{t}^{(s(t, T))}\right\}
$$

where $s: \mathbb{N} \times \mathbb{R} \rightarrow \mathcal{I}$ is a static function that maps the time sample $t$, and the switching period $T$ to an element of the index set $\mathcal{I}$ is defined as follows:

$$
s(t, T)=\left\lfloor\frac{1}{T} \bmod (t, N T)\right\rfloor
$$

where $\bmod (x, y)$ is the modulo operator, and $\lfloor\cdot\rfloor$ is the floor function.

By using the proposed watermark (cf. Eq. 10), we now have an adaptive protection mechanism with two main configurable parameters: the number of distributions $N$ and the switching frequency $f=1 / T$. It is worth to notice that the original watermark signal described in Section 3.2 is recovered when $f \rightarrow 0$ and when $\Delta U^{(0)}$ being a stationary zero mean Gaussian process.

\subsection{Single-watermark LQG structure performance loss}

In this section, we compute the increment of cost in the LQG structure due to the single-watermark added to the control input. This supplementary cost is the degradation in the performance of the system, as shown in [5], and can be defined as follows:

$$
J=J^{*}+\Delta J_{s}
$$

where $J^{*}$ is the optimal cost of the system described in Section 3.1; and $\Delta J_{s}$ is the increment of cost due to the use of the single-watermark-based detector. In the following, we develop the cost of the system in the time domain.

$$
\begin{aligned}
J & =\lim _{n \rightarrow \infty} \frac{1}{n} \sum_{j=0}^{n-1} E\left[\left(x_{j}^{T} \Gamma x_{j}+u_{j}^{T} \Omega u_{j}\right)\right] \\
& =\lim _{n \rightarrow \infty} \frac{1}{n} \sum_{j=0}^{n-1}\left[\operatorname{tr}\left(\Gamma \operatorname{Cov}\left(x_{j}\right)\right)+\operatorname{tr}\left(\Omega \operatorname{Cov}\left(u_{j}\right)\right)\right]
\end{aligned}
$$

where $x_{t}$ is defined as:

$$
x_{t}=\mathcal{L}_{x}\left(w_{t}, v_{t}\right)+\sum_{j=t-1}^{\infty}(A+\mathrm{BL})^{j} B \operatorname{Var}\left(\Delta u_{-j}\right)
$$


and $u_{t}$ as follows:

$$
u_{t}=\mathcal{L}_{u}\left(w_{t}, v_{t}\right)+\sum_{j=t-1}^{\infty}(A+\mathrm{BL})^{j} B \operatorname{Var}\left(\Delta u_{-j}\right)+V \operatorname{ar}\left(\Delta u_{t}\right)
$$

where $\mathcal{L}_{x}$ and $\mathcal{L}_{u}$ are linear functions. Their definition is not relevant for the target of this paper, but the reader can find the details in [5]. Assuming that the noise of the system and the watermark are independents, we can define the increment of cost due to the single-watermark as [5]:

$$
\begin{aligned}
\Delta J_{s}= & \Gamma \operatorname{tr}\left[\operatorname{Cov}\left(\sum_{j=t-1}^{\infty}(A+\mathrm{BL})^{j} B \operatorname{Var}\left(\Delta u_{-j}\right)\right)\right] \\
& -L \Omega \operatorname{tr}\left[\operatorname{Cov}\left(\sum_{j=t-1}^{\infty}(A+\mathrm{BL})^{j} B \operatorname{Var}\left(\Delta u_{-j}\right)+\operatorname{Var}\left(\Delta u_{t}\right)\right)\right]
\end{aligned}
$$

\subsection{Multi-watermark LQG structure performance loss}

Let us now evaluate the increment of the cost generated by the multi-watermark-based detector, $\Delta J_{m}$, and next, compare the cost generated by the single- and the multi-watermarks. The equation of $\Delta J_{m}$, is given by

$$
\begin{aligned}
\Delta J_{m}(t)= & \operatorname{tr}\left[\Gamma \operatorname{Cov}\left(\sum_{j=t-1}^{\infty}(A+\mathrm{BL})^{j} B\left(\operatorname{Var}\left(\Delta u_{-j}^{(i)}\right)+E\left[\Delta u_{-j}^{(i)}\right]\right)\right)\right] \\
& +\operatorname{tr}\left[L \Omega \operatorname { C o v } \left(\sum_{j=t-1}^{\infty}(A+\operatorname{BL})^{j} B\left(\operatorname{Var}\left(\Delta u_{-j}^{(i)}\right)+E\left[\Delta u_{-j}^{(i)}\right]\right)\right.\right. \\
& \left.+\left(\operatorname{Var}\left(\Delta u_{t}\right)+E\left[\Delta u_{t}\right]\right)\right]
\end{aligned}
$$

where $\operatorname{Var}\left(\Delta u_{t}^{(i)}\right)$ and $E\left[\Delta u_{t}^{(i)}\right]$ are, respectively, the variance and the mean of the watermark sent at moment $t$. The performance loss of the LQG structure depends linearly on the variance and the mean of the multiwatermark $\Delta u_{t}^{(i)}$ for each $T$ samples.

The following theorem shows the difference between the performance loss due to the single-watermark, $\Delta u$, and the performance loss due to the multi-watermark, $\Delta u^{(i)}$.

Theorem 4.1 Let us assume that a watermark is a Gaussian signal with a couple of parameters to be characterized; the mean and the variance. The multi-watermark distribution is defined as $M_{w}=N\left(E\left[\Delta u^{(i)}\right], \operatorname{Var}\left(\Delta u^{(i)}\right)\right)$, and the single-watermark distribution is defined as $S_{w}=$ $N(E[\Delta u], \operatorname{Var}(\Delta u))$. If we define for the multi-watermark $\beta$ as:

$$
\beta=E\left[\Delta u^{(i)}\right]+\operatorname{Var}\left(\Delta u^{(i)}\right) \quad \forall i \in \mathcal{I}
$$

and for the single-watermark $\epsilon$ as:

$$
\epsilon=E[\Delta u]+\operatorname{Var}(\Delta u)
$$

where $\epsilon$ and $\beta$ are constant for single- and multiwatermarks, respectively. Then, we can conclude that the performance loss of both approaches is equal if $\epsilon=\beta$.

Proof If we assume that $E[\Delta u]=0$ for the singlewatermark, we can prove the theorem as follows:

$$
\begin{aligned}
\operatorname{Diff}\left(\Delta J_{m}(t), \Delta J_{s}(t)\right)= & \Delta J_{m}(t)-\Delta J_{s}(t) \\
= & \Gamma \operatorname{tr}\left[\operatorname{Cov}\left(\sum_{j=t-1}^{\infty}(A+\mathrm{BL})^{j} B\left(\beta_{-j}-\epsilon_{-j}\right)\right)\right] \\
& +L \Omega t r\left[\operatorname { C o v } \left(\sum_{j=t-1}^{\infty}(A+\mathrm{BL})^{j} B\left(\beta_{-j}-\epsilon_{-j}\right)\right.\right. \\
& \left.\left.+\left(\beta_{t}-\epsilon_{t}\right)\right)\right]=0
\end{aligned}
$$

Remark 4.2 Note that using Theorem 4.1, the performance loss due to the multi and the single-watermark is equal. The assumption of the equal performance loss allows to compare both approaches under the same conditions. This can be formally stated as follows:

$$
E\left[\Delta u^{(i)}\right]+\operatorname{Var}\left(\Delta u^{(i)}\right)=\epsilon=\beta
$$

\subsection{Numerical validation of the multi-watermark detector against non-parametric cyber-physical adversaries}

This section validates through numerical simulations the detection scheme proposed in Section 4.1. In particular, we aim at showing that the proposed watermark signal is able to detect non-parametric cyber-physical adversaries (cf. Section 3.3) with a higher detection ratio with respect to the one obtained with the watermark proposed in $[4,5]$. Toward this end, we employ a MIMO system with four inputs and four outputs described by the following matrices: 


$$
\begin{aligned}
& A=\left[\begin{array}{ccccccc}
0.3991 & 0.07113 & 0.1573 & -0.1274 & 0.0226 & -0.0225 & 0.001 \\
0.003 & -0.07588 & -0.005092 & -0.03893 & 0.09917 & -0.0168 & 0 \\
-0.1974 & -0.01849 & 0.0453 & 0.1579 & -0.1597 & 0.1405 & -0.002 \\
-0.1246 & -0.0726 & 0.1515 & -0.1148 & 0.5156 & -0.0665 & 0 \\
0.4309 & -0.1204 & 0.09715 & 0.055 & 0.2406 & 0.2812 & 0.0001 \\
-0.0827 & -0.01092 & 0.1234 & -0.1318 & 0.0348 & 0.469 & 0 \\
0.08312 & -0.0829 & 0.081 & 0.0358 & 0.1124 & 0.02475 & 0.4469
\end{array}\right] \\
& B=\left[\begin{array}{cccc}
0.947 & 0 & 0.002 & -0.021 \\
-0.0086 & 0 & -0.406 & 0.02829 \\
-0.8708 & 0.0011 & 0.0011 & -0.106 \\
0.4872 & 0.002 & 0.188 & -0.041 \\
0.1233 & 0 & 0.01 & -0.9344 \\
0 & 0 & 0 & 0.521 \\
0 & 0.7658 & 0 & 0
\end{array}\right] \\
& C=\left[\begin{array}{ccccccc}
-1.102 & 0.302 & -0.1004 & 0.0386 & 0.053 & 0.0891 & 0 \\
0 & 0.114 & -0.0132 & -1.087 & 0.116 & 0.051 & 0.905 \\
0.0003 & 1.593 & -0.002 & 0 & 0.093 & 0 & 0.0428 \\
-0.163 & -0.0712 & -0.1074 & 0 & 0 & -0.7443 & 0.089
\end{array}\right] \text {. }
\end{aligned}
$$

and co-variance matrices equal to $Q=0.2 I$ and $R=I$. The positive definite cost matrices $\Gamma$ and $\Omega$ are both equal to the identity matrix. The simulation is based on MATLAB and Simulink models of the plant, as well as the models of the non-parametric cyber-physical adversaries. The attacks start at $t=700 \mathrm{~s}$. We use three different distributions (i.e., $N=3$ ) switched at random; a Gaussian, a Rician, and a Rayleigh distribution. Table 2 shows the covariance and offset configured in the simulations for each distribution.

To validate the proposed attack-detection scheme, we compare the system dynamics considering two different switching frequencies. We have simulated a high frequency switching watermark configured to switch each 7 time samples, and a low frequency switching configured to switch each 20 time samples. Figure 5a, c shows the plant dynamics and the dynamics of the states estimated by the controller in the case of a switching frequency watermark configured to 7 time samples and a cyber-physical adversary attack. Figure 5a shows that the adversary is able to drive the state to an undesired value. Nevertheless, the controller misled by the adversary, does not perceive such situation (cf. Fig. 5c). Figure 5b, d shows the plant dynamics and

Table 2 Sample parameters used in the multi-watermark MATLAB/Simulink implementation

\begin{tabular}{lll}
\hline Distribution & Variance $\left(\sigma^{2}\right)$ & Offset \\
\hline Gaussian & 5.9536 & 0.0 \\
Rician & 3.8870 & 3.7106 \\
Rayleigh & 3.0581 & 2.5553 \\
\hline
\end{tabular}

the dynamics of the state estimated by the controller when the watermark is switched each 20 time samples. The dynamics show exactly the same behavior described above.

Figure 5e, $\mathrm{f}$ shows the dynamics of the alarm signal $g_{t}$ produced by the detector, respectively, in the case of high and low switching frequencies. Notice that switching the watermark distributions at a high frequency provides better detection performances compared to the case of a low switching frequency.

To quantify the effectiveness of the proposed detection scheme, we compute the detection ratio DR as a function of the switching frequency. In particular, for each considered frequency $f$ we run 200 Monte Carlo simulations (with randomly generated system parameters) both in the case of the cyber-physical and the cyber adversary, and we compute the CDF (cumulative distribution function) of the detection ratio.

We start by confronting the performance obtained with the detection strategy based on multiple watermark signals proposed in this paper with that proposed in $[4,5]$ in both the case of a cyber-physical and a cyber adversary. In the case of the proposed multi-watermark strategy, we consider two switching frequencies $f_{L}=0.05 \mathrm{~Hz}$ (switching watermark each 20 timesteps) and $f_{H}=0.14 \mathrm{~Hz}$ (switching watermark each 7 timesteps). The results of this comparison are shown in Fig. 6. Let us focus on the detection strategy proposed in $[4,5]$ : as shown before, the detector is able to consistently detect a cyber attack, but it performs poorly when a cyber-physical adversary attacks the system. Nevertheless, the proposed detection strategy based on multiple watermarks is able to provide a higher detection ratio. In 


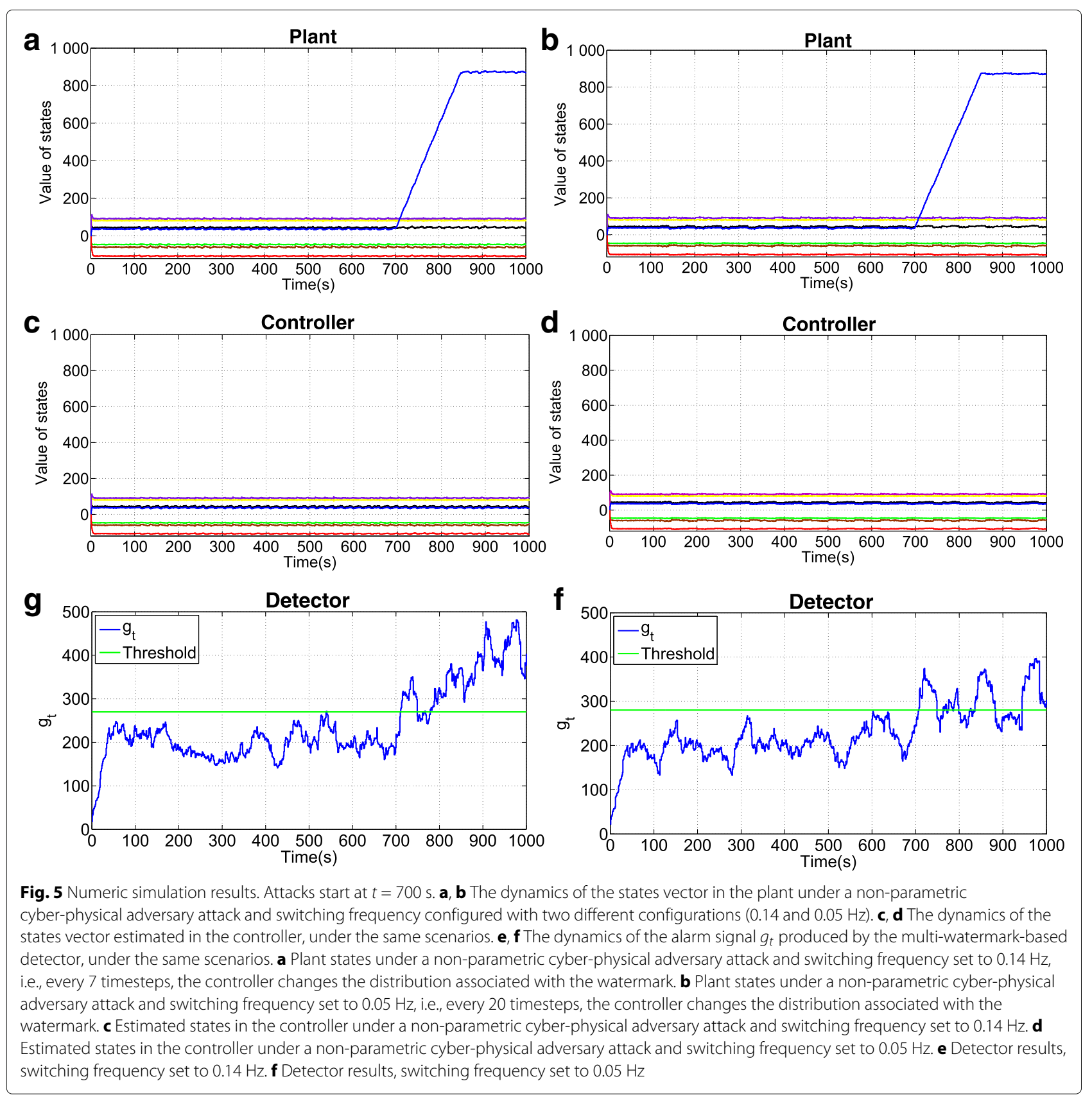

particular, we notice that the detector employing a higher switching frequency $f_{H}$ provides better performances with respect to the case of using the lower switching frequency $f_{L}$.

In the following, we are interested in analyzing in more details the performance of the proposed detection strategy, when the switching frequency $f$ is varied, to give a more in depth explanation of the anecdotal evidence shown above. Toward this end, Fig. 7a shows the CDF of the detection ratio obtained when the switching frequency varies in the range $0.10-0.33 \mathrm{~Hz}$. In this case, we only consider cyber-physical adversaries. The CDFs shown in Fig. 7a confirm that when the switching frequency increases, the detection ratio also increases.

To finish this section, we provide in Fig. 7b the median detection ratio function of $f$. The figure also contains the case $f=0$ that corresponds to the detection strategy proposed in [4, 5] - used as a baseline. The shaded area in the figure corresponds to the values of the detection ratio between the 25 and the $75 \%$ for each $f$. As expected, the figure shows that by increasing the frequency, we are 


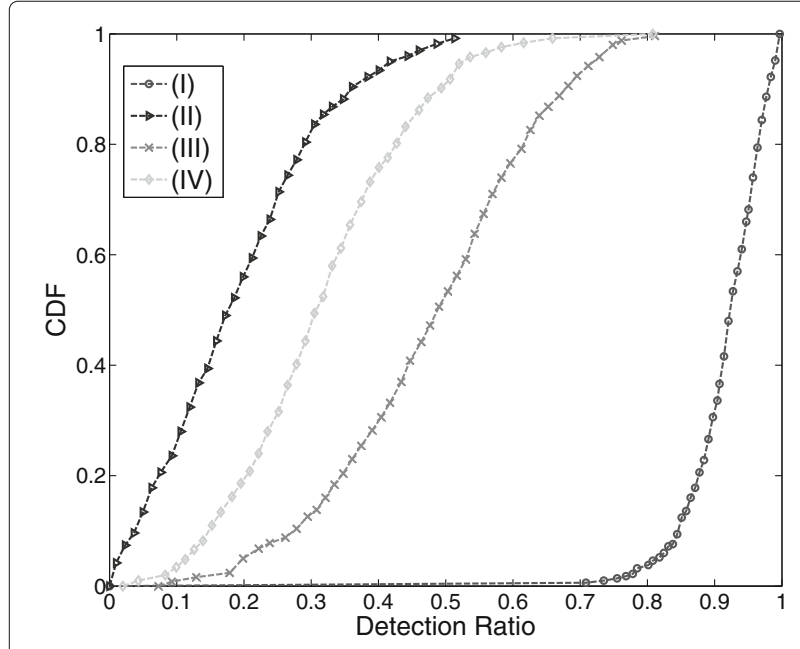

Fig. 6 Numeric simulation results using the single- and multi-watermark detection schemes. Confronting the performance of the two detectors. (I) $\chi^{2}$ detector in $[4,5]$ and cyber adversary. (II) $\chi^{2}$ detector and non-parametric cyber-physical adversary. (III) Multi-watermark detector with switching frequency set to $0.14 \mathrm{~Hz}$ and non-parametric cyber-physical adversary. (IV) Multi-watermark detector with switching frequency set to $0.05 \mathrm{~Hz}$ and non-parametric cyber-physical adversary

able to obtain a detection ratio that goes from around 0.2 in the case of the baseline approach of $[4,5]$ to around 0.6 in correspondence of $f=0.33 \mathrm{~Hz}$.

Observe that the probability of false alarms without attack (often referred in the related literature as false positives) is fixed, $\alpha=1 \%$. Notice as well that false negatives (i.e., undetected real attacks) are inversely proportional to the detection ratio for each switching frequency.

\subsection{Efficiency validation}

We have validated above the multi-watermark detector using a static function, $\mathcal{I}$, to define the multi-watermark and different performance loss between single- and multiwatermarks. We next present the results and validations obtained for a system with the same performance loss between single- and multi-watermark detectors and where the multi-watermark is generated from a nonstatic function, $\mathcal{I}_{d}$. Figure 8 shows the result obtained after running 200 Monte Carlo simulations of a system with single- and multi-watermark detectors against a nonparametric cyber-physical adversary. In this simulation, single- and multi-watermark detectors have 30\% performance loss, $\Delta J$, with respect to the optimal cost. Moreover, the watermark uses a dynamic function to define the multi-watermark. In Fig. 8a, we show the result of using both single- and multi-watermark for a system of order four. We can confirm that the multi-watermark detector, with the same performance loss as the single-watermark detector, has a higher detection ratio. Figure $8 \mathrm{~b}$ shows the result of single- and multi-watermarks for a higher system of order, 25. On the one hand, these results confirm that the multi-watermark detector is able to detect properly non-parametric cyber-physical adversaries. Additionally, we can conclude that the detection ratio increases with the complexity of the system. On the other hand, Fig. 9 depicts that using the multi-watermark approach, with same performance loss as in the case of using only the
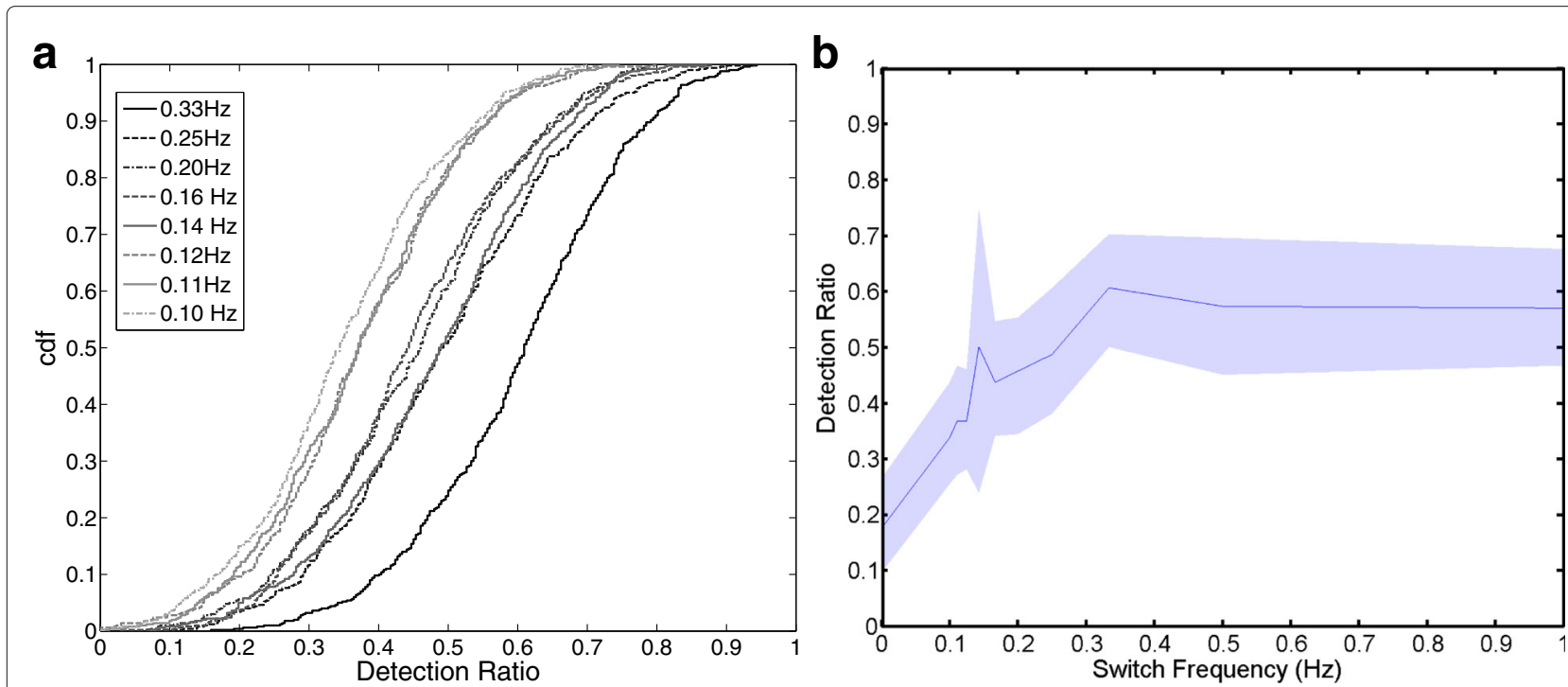

Fig. 7 Numeric simulation results using the multi-watermark detection scheme. a Cumulative distribution function (CDF) of the detector under different switching frequencies. b Median detection ratio function per switching frequency, in which the shaded area corresponds to the 25 and the $75 \%$ (i.e., confidence intervals). a CDF and detection ratio per switching frequency. $\mathbf{b}$ Detection ratio function with respect to the switching frequency 


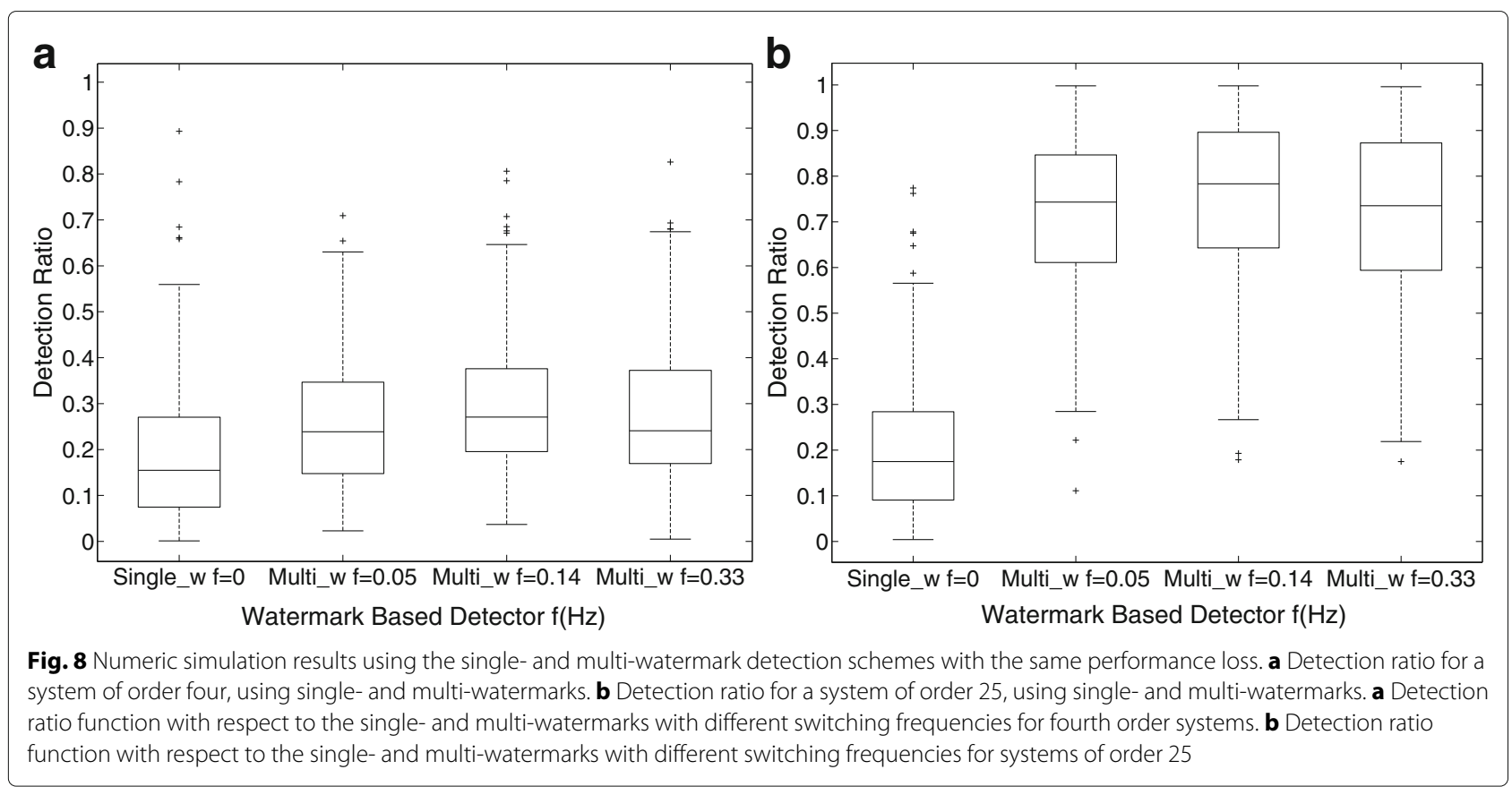

single-watermark, the detection ratio increases when the switching frequency varies in the range $0-0.14 \mathrm{~Hz}$, where $f=0$ is the single-watermark detector. In the following section, we extend the analysis to the case of parametric cyber-physical adversaries.

\subsection{Numerical validation of the multi-watermark detector against parametric cyber-physical adversaries}

In the previous sections, we have seen how the multiwatermark detector is able to detect both cyber and nonparametric cyber-physical adversaries. In this section, we extend the study to the case of parametric cyber-physical adversaries (cf. Definition 3.6). We recall that parametric cyber-physical adversaries are able to identify the system model parameters from the input and the output plant signals. In fact, a parametric cyber-physical adversary can obtain the system model with great accuracy, if control commands and sensor measurements are accessible.

Let us first show how a parametric cyber-physical adversary acquires the watermark signal presented in $[4,5]$. Remember that such a watermark is modeled as a

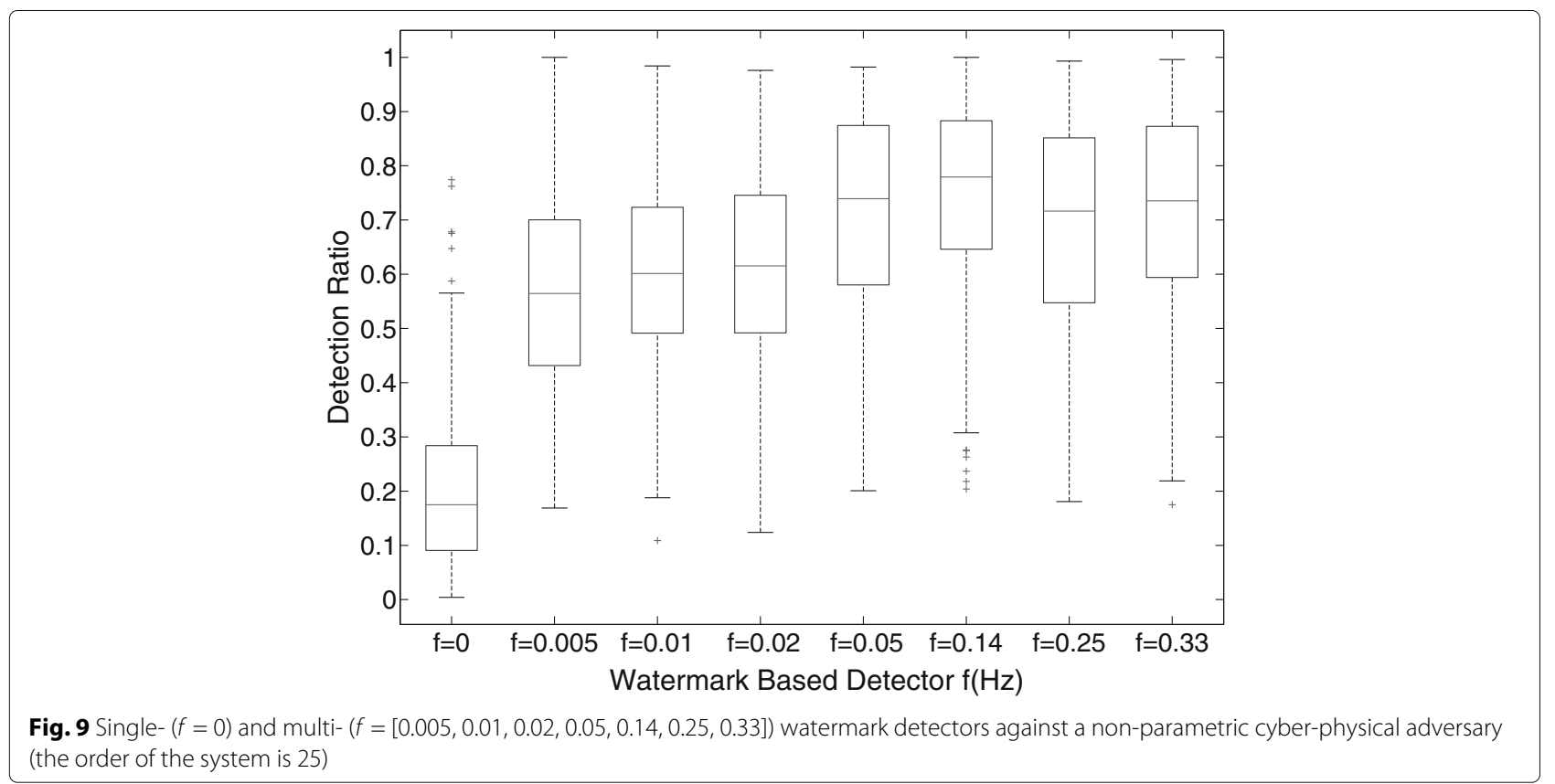


Gaussian signal with zero mean. Its variance is represented by $\mathcal{U}$, i.e., $\Delta u_{t} \sim N(0, \mathcal{U})$. The variance modifies the control inputs and propagates the modification to the system outputs. However, it does not modify the system dynamics. Control inputs are represented by

$$
U(z)=U^{*}(z)+\Delta U(z)
$$

and the system outputs are represented by

$$
\begin{aligned}
Y(z) & =H(z)(U(z)+W(z))+V(z) \\
& =H(z)\left(U^{*}(z)+\Delta U(z)+W(z)\right)+V(z)
\end{aligned}
$$

Using the aforementioned characteristic of the watermark, a parametric cyber-physical adversary can use an ARX (autoregressive with exogenous input) model to define the system as follows [22]:

$$
Y(z)=H(z) U(z)+V(z)
$$

where $U(z)$ and $Y(z)$ represent the inputs and the outputs of the plant, respectively; $V(z)$ represents the external noise which affects the outputs of the plant; and $H(z)$ is another way to describe the model of the system presented in Section 3, such that:

$$
H(z)=\frac{\mathcal{N}(z)}{\mathcal{D}(z)}=\left(\frac{n_{0} z^{m}+n_{1} z^{m-1}+\ldots+n_{m}}{d_{0} z^{n}+d_{1} z^{n-1}+\ldots+d_{n}}\right)
$$

where $\mathcal{N}(z)$ and $\mathcal{D}(z)$ are the polynomial functions which build the model of the system.
Following the same simulation setup introduced in Section 4.4, Figs. 10 and 11 show the detection ratio of the watermark detector against a parametric cyberphysical adversary. Figure 10 shows the results of 200 Monte Carlo simulations using systems of order 10 against this adversary. The results present the detection ratio if the adversary uses a window size equal to 200 and different system orders for the model. If the adversary order varies in the range $[8,12]$, the detection ratio is not higher than $10 \%$. Out of this range, the detection ratio increases drastically. Figure 11 shows the detection ratio using systems of order 25, against seven different parametric cyber-physical adversaries. The assumed window size is settled to $\hat{T}=300$. The range of orders where the detection ratio does not increase drastically is $[18,28]$. If an adversary uses an order in this range, the detection ratio is not higher than $10 \%$. Otherwise, the likelihood to detect the adversary is high.

Figure 12 shows the detection ratio of the same system, against a parametric cyber-physical adversary with different window sizes $(125,150,200,250$, and 300), and the correct system order. It is worth noting that the adversary needs a bigger window size in order to attack a system using a higher order, with a detection ratio less than $10 \%$. Following the previous results, we can conclude that a parametric cyber-physical adversary, who is capable to eavesdrop and analyze a large number of samples from the communication channel and use an equivalent order system, is capable of evading detection.
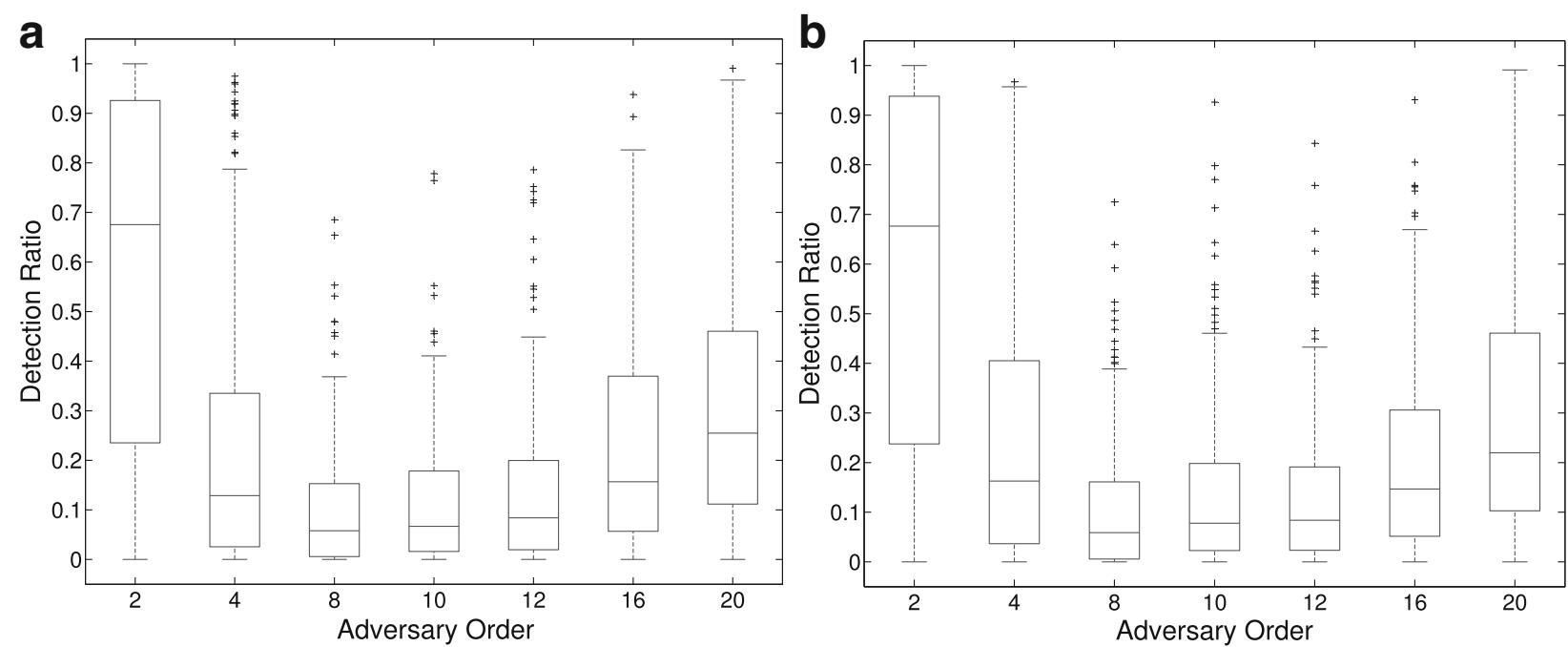

Fig. 10 Numeric simulation results using the single- and multi-watermark detection schemes with the same performance loss, different adversary system order, and a window size equal to 200. a Detection ratio regarding single-watermark for systems of order ten. $\mathbf{b}$ Detection ratio regarding multi-watermark for systems of order ten. a Detection ratio function with respect to the single-watermark against a parametric cyber-physical adversary using different adversary system order. b Detection ratio function with respect to the multi-watermark against a parametric cyber-physical adversary using different adversary system order 


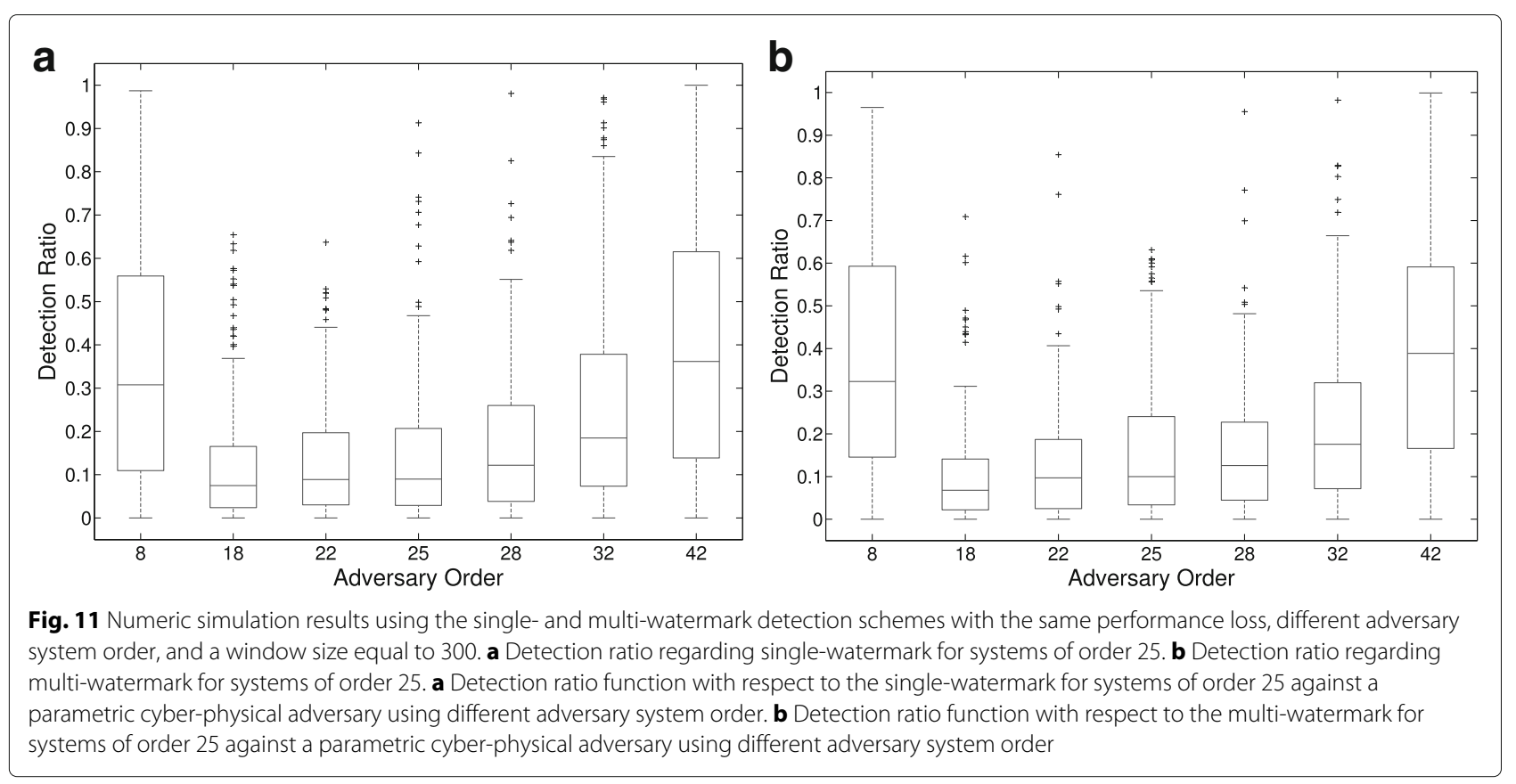

Remark 4.3 A parametric cyber-physical adversary is able to obtain the system model, $H(z)$, and mislead the controller, by eavesdropping the control inputs and the sensor measurements. The probability of being detected is equivalent to the probability of obtaining an erroneous model. This probability is directly proportional to the order of the system and inversely proportional to the window size to eavesdrop the data channel.
Following the Remark 4.3, and under the hypothesis of considering the real system like a black box, erroneous system identification depends on the order selected by the adversary to recreate the system model, as well as the number of eavesdropped samples and the window size used by the adversary to recompute the parameters of the target system. This situation can be quantified using mean squared error (MSE) [26, 27]. In a nutshell,

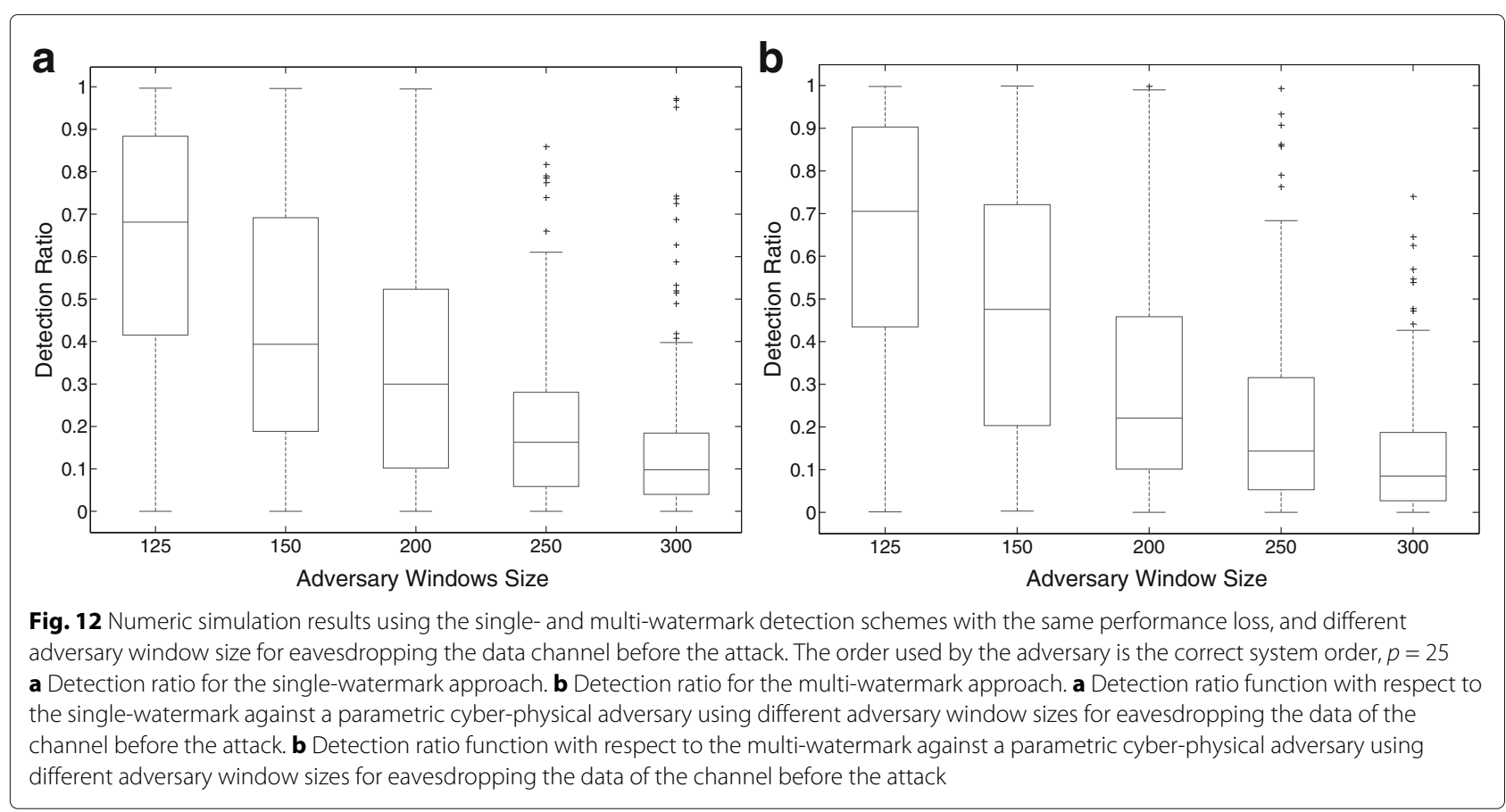


the likelihood to obtain the correct model of the target system is directly proportional to the order chosen by the adversary to generate the model, and inversely proportional to the number of samples eavesdropped (cf. Figs. 10-12). The computational cost for the adversary is directly proportional to the system order, since the adversary needs to increase the order of the model, as well as the window size in order to minimize the MSE. Therefore, the number of samples eavesdropped before conducting the attack, together with the order system chosen by the adversary, are the two main parameters to evade detection.

\section{Experimental results with a laboratory SCADA testbed}

In this section, we present some experimental results obtained with a real-world implementation of the detection mechanisms and adversary models presented in this paper. The implementation is conducted using a laboratory testbed based on SCADA protocols such as Modbus and DNP3 (cf. Section 2.1).

\subsection{Testbed design}

The architecture proposed for our SCADA testbed works as follows: all the elements (controller, sensors, actuators, PLCs, and RTUs) can be distributed across several nodes in a shared network combining DNP3 and Modbus protocols (cf. Fig. 13). Likewise, one or various elements can be embedded into a single device. From a software standpoint, the controller never connects directly to the sensors. Instead, it is integrated in the architecture as a SCADA PLC node, with eventual connections to some other intermediary nodes. Such nodes are able to translate the controller commands into SCADA (e.g., either Modbus or DNP3) commands. As depicted in Fig. 13, the architecture is able to handle several industrial protocols and able to connect to complementary SCADA elements, such as additional PLCs and RTUs. To evolve the architecture into a complete testbed, new elements can be included in the system, such as additional proxy-like RTU nodes.

From a data transmission standpoint, we include in our SCADA testbed the possibility of using different sampling frequencies, in order to cover a larger number of experimental scenarios. The architecture is able to handle several PLCs. To avoid overloading one channel with all the possible registers of the PLCs, separate ports are designated in order to isolate the communication between separated PLCs. DNP3 commands perform an Integrity Scan which gathers all the data from the PLCs in case several PLCs were being handled in the same channel, all variables of the a PLC would be fetched causing overhead in the communication.

\subsection{Experimentation}

We present in this section the results of applying the watermark authentication techniques proposed in this paper. Several repetitions of the experiments are orchestrated using automated scripts handling the elements of some representative scenarios. A set of attacks and detectors are used and posteriorly analyzed. The combinations, attack-detector, are the following:

- Replay attack-watermark disabled. In this scenario, the attacker is likely to evade the detector, since no watermark is injected into the system.

- Replay attack-watermark enabled. In this scenario, the attacker is likely identified by the detector, since the attack is not able to adapt to the current watermark.

- Non-parametric attack-stationary watermark. Using this scenario, the attacker and the detector have equal chances of success.

- Non-parametric attack-non-stationary watermark. Using this scenario, the non-stationary watermark changes the distribution systematically, hence, preventing the attack to adapt to such changes. The expected results are an increase of the detection ratio.

- Parametric attack-stationary watermark. In this scenario, the attacker is likely to evade the detector when the attack properly infers the system parameters.

- Parametric attack-non-stationary watermark. The attacker is also likely to evade the detector when the system parameters are properly identified.

The cyber-physical implications of the testbed hinder the experimentation process especially when several repetitions are required in order to obtain statistical results, contrary to simulations where only the code is executed. The creation of the orchestration script, which automates the test, is necessary to simplify the experimentation tasks. The next section presents the results using the testbed for the aforementioned attacker-detector combinations. Some sample executions of the replay attackwatermark disabled scenario, as well as information about the implemented techniques, are available at http://j.mp/ TSPScada.

\subsection{Experimention and results}

After collecting data from different devices across the SCADA testbed, the data is analyzed accordingly to interpret the performance of the detector with regard to the attack scenario. Since the stationary watermark detector was correctly refined for each test scenario, we are able to analyze in depth the results through a statistical evaluation of the data. Experimental results with the nonstationary watermark mechanism are also conducted. 


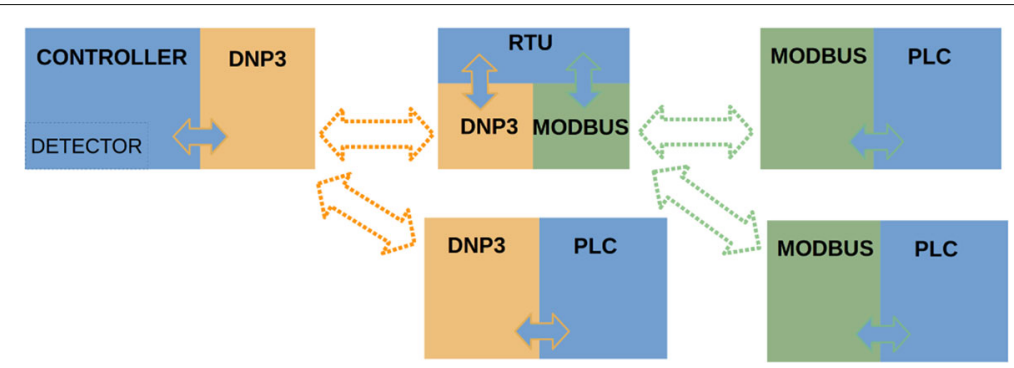

Fig. 13 Abstract architecture overview

Figure 14 shows the detector values, $g_{t}$, for all the attackdetector combinations defined in Section 5.2.

For all the plots, the solid horizontal line represents the threshold, and the vertical dotted line represents the moment when the attacker starts injecting malicious data. The short peaks on the left side of the plots, those bypassing the threshold line before the start of the attacks, are counted as false positives or system faults.

Figure 14a, b shows the experimental results of the replay attack. When the watermark is disabled (cf. Fig. 14a), the attacker properly evades the detector. Since the controller is not inserting the protection watermark, it does not detect the attack. On the contrary, the results in Fig. 14b show that the activation of the watermark under the same scenario allows the controller to alert about the attack almost immediately. Based on these results, we can conclude that the stationary watermarkbased detector properly works out to detect the replay attack.

Figure 14c represents the non-parametric attacker against the previously tested stationary watermark. The detector is now unable to detect the attacker. Figure 14d shows the case where the non-stationary watermark is enabled. Under this situation, the detector has lightly more chances of detecting the attack. This shows how the non-stationary watermark mechanism does improve the detection abilities compared to the stationary watermark approach.

Figure 14e, f evaluates the scenario associated with the parametric attacks. Theoretically, the attacker is expected to evade the detector when the attack succeeds at properly identifying the parameters of the system dynamics. Figure 14e represents the experiments where the parametric attack is executed under the stationary watermark scenario. The figure shows that the detector value, $g_{t}$, remains most of the time below the detection threshold. Figure 14f shows the behavior of the detector under the non-stationary watermark scenario. This time, the detector has slightly more chances of detecting the attack.

\subsection{Statistical data evaluation}

Using the watermark-based detection mechanism, we run for each attack scenario 75 automated rounds (about $4 \mathrm{~h}$ of data collection processing). In order to evaluate the results, we use the following metrics:

1. Detection ratio, associated with the success percentage of the detector, calculated with regard to the time range after each attack starts.

2. Average detection time, determining the amount of time needed by the detector to trigger the attack alert.

3. False negative (FN) ratio, determining the number of samples where the detector fails at successfully alerting about the attacks. The ratio is calculated as follows:

$$
F N=\frac{S A-A D}{S A}
$$

where $S A$ represents the values of the samples under attack, and $A D$ the samples detected as an attack.

4. False positives (FP) ratio, calculated as the number of samples where the detector signals benign events as attacks. The ratio is calculated as follows:

$$
\mathrm{FP}=\frac{\mathrm{AD}}{\mathrm{SN}}
$$

where $S N$ represents the number of samples under normal operation, and $A D$ the number of samples detected as attack by mistake.

Table 3 shows the performance results of the detector, based on the detection ratio and the average detection time metrics.

Regarding the results shown in Table 3, we can emphasize that the replay attack is the most detectable scenario, with a detection ratio of about $40 \%$. This detection

Table 3 Detector performance results

\begin{tabular}{llll}
\hline & $\begin{array}{l}\text { Replay } \\
\text { attack }\end{array}$ & $\begin{array}{l}\text { Non-parametric } \\
\text { attack }\end{array}$ & $\begin{array}{l}\text { Parametric } \\
\text { attack }\end{array}$ \\
\hline Detection ratio & $40.00 \%$ & $18.00 \%$ & $12.00 \%$ \\
Average detection time & $18.81 \mathrm{~s}$ & $10.17 \mathrm{~s}$ & $6.08 \mathrm{~s}$ \\
\hline
\end{tabular}




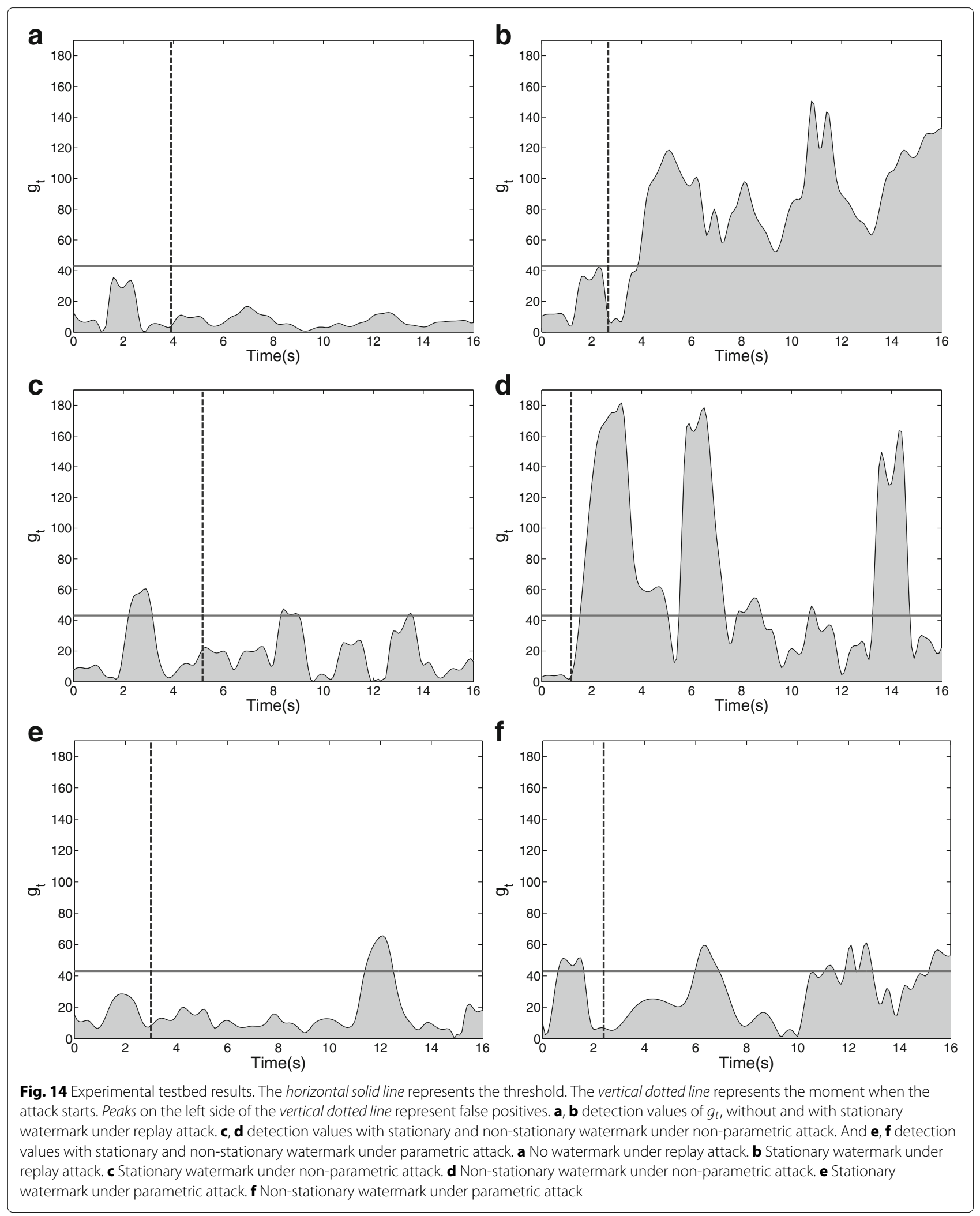


ratio is still far from being perfect, which we ascribe to imperfect sensors accuracy and resolution. The nonparametric attacker has a lower detection ratio, of about $18 \%$. This result is expected, as suggested by the theoretical and simulation-based conclusions presented in Sections 3 and 4. The parametric attack has the most robust system identification approach. The attacks can evade the detection process if they succeed at properly identifying the system attributes. In terms of results, they lead to the lowest detection rate of about $12 \%$.

During the replay attack, the average detection time is the slowest of all the adversarial scenarios. This behavior is due to the watermark distribution properties, since the watermark variation makes the replay attack highly detectable. At the same time, the injection attacks (either the parametric or the non-parametric version) are detected much faster than the replay attack. This is due to the transition period needed by the attackers to estimate the correct data prior misleading the detector. For this reason, if the attacker does not choose the precise moment to start the attack, the detector implemented at the controller side is able to detect the injected data, right at the beginning of the attack. Furthermore, the attackers shall also synchronize their estimations to the measurements sent by the sensors. In the case the synchronization process fails, the detector identifies the uncorrelated data and reports the attack.

Table 4 shows that the detection of the replay attack has the lowest false negative ratio, $64.06 \%$, hence, confirming that this adversarial scenario is the most detectable situation with regard to the detection techniques reported in $[4,5]$. The detection of the non-parametric attacks has a higher false negative ratio, $85.20 \%$, confirming the theoretical and simulation-based results reported in Section 4. The detection of the parametric attacks also confirms the results obtained via numeric simulations, leading to the highest false negative ratio (about 88.63\%). In terms of false positive ratio, the three adversarial scenarios show a very low impact of our detection approach (on average, about $1.33 \%$ false positive ratio).

\section{Discussion}

In Section 3, we have reviewed the watermark-based detector proposed in $[4,5]$. We have shown that the detector fails at properly handling attacks carried out by cyber-physical adversaries. In particular, we have shown that an adversary that learns about the system model is

Table 4 Long run experimental results

\begin{tabular}{llll}
\hline & Replay attack & Non-parametric attack & Parametric attack \\
\hline False negatives & $64.06 \%$ & $85.20 \%$ & $88.63 \%$ \\
False positives & $0.98 \%$ & $1.66 \%$ & $1.35 \%$ \\
\hline
\end{tabular}

able to separate the watermark from the control signal and able to succeed at attacking the system without being detected. Then, we have presented an enhanced detection scheme. The main idea of the new scheme is to use multiple watermark distributions and non-stationary identification signals. The resulting approach is able to detect both cyber- and cyber-physical adversaries.

To summarize, the detector in $[4,5]$ fails at detecting cyber-physical adversaries. The multi-watermark proposal succeeds at properly detecting such adversaries under the assumption that the watermark distributions change quite frequently. The rationale is that, the nonparametric adversary has little chances of acquiring the necessary information to acquire the watermark and bypass the detector. Moreover, the detector performance loss in the multi-watermark approach is equivalent to the performance loss in the case of the single-watermark approach. We have also shown that a smarter parametric cyber-physical adversary is able to attack the system without being detected in case of detecting the correct parameters. We have detailed the strategy of this adversary in Section 4. It is worth noting that the detection ratio increases with respect to the lack of accuracy of the adversary.

\section{Related work}

Security of cyber-physical systems (CPSs) is drawing a great deal of attention recently [1] after the infamous StuxNet malware [2] uncovered the potential of successful security attacks carried out against such systems.

In the following, we separately overview some related work in the literature of CPS security by focusing on three main research areas: (1) security requirements and threat analysis; (2) control-theoretic solutions for the detection of cyber-physical attacks; and (3) implementation of experimental testbeds.

\section{Security requirements and threat analysis on CPSs} Several authors have studied the requirements to take into account the new security issues when designing security mechanisms for cyber-physical systems. In [28], Cardenas et al. define the issue of secure control by analyzing separately the problem first from an information security point of view and then by looking at specific control issues. In [29], the same authors outline for the first time the difference between corporate ICT security and cyberphysical system security, and the importance of explicitly considering the interactions of the control system with the plant. In [30], Wang et al. provide a classification of threats and possible attacks against CPS. They also introduce a taxonomy of adversaries by classifying them into several categories depending on skills and motivation. A different perspective is taken in [15] where Texeira et al. propose to classify attacks according to a three-dimensional 
cyber-physical attack space; disruption of assets, disclosure of resources, and adversary knowledge. Cyber attacks specific to SCADA systems are addressed in [31, 32]. In [31], Ten et al. carry out a quantitative assessment of vulnerability of SCADA systems. Zhu et al. analyze in [32] some attacks toward SCADA systems by making a distinction between hardware, software, and communication stack threats. In a complementary research direction, the issue of designing testbeds to experimentally evaluate both threats (and their countermeasures) has been carried out by Hahn et al. and Mallouhi et al. in $[33,34]$.

Control-theoretic solutions for the detection of cyberphysical attacks This research line explicitly considers the interconnection between cyber and physical control domains in networked-control systems. Recently, the control system community started to study security of cyberphysical systems both under the methodological point of view and from a more technological standpoint by looking at particular problems arising in, e.g., smart grids, power grids, and water distribution systems. Concerning the methodological aspects, several studies have proposed to adapt classical frameworks (developed to cope with model uncertainties and fault diagnosis) to handle security issues in networked-control systems. Table 5 gathers the cyber-physical attacks handled in the literature. Among these cyber-physical attacks, the replay attack is the only one in which the adversary is able to carry out actions without knowledge about system model dynamics. To carry out the remainder attacks, the adversary requires complete knowledge about the dynamics of the system. For example, to execute the dynamic false-data injection attack handled by Mo et al. in [35], the adversary has to have a perfect knowledge of the plant's behavior. To execute a covert attack, handled by Smith et al. [16], it is necessary to have knowledge of the plant's and controller's behavior. Concerning detection mechanisms, several lines of research consider the adaptation of fault detection systems to detect intentional attacks $[5,15,36]$. Mo et al. show in [5] that it is possible to detect replay attacks by properly watermarking control inputs. Pasqualetti et al. propose in [36] to employ geometric

Table 5 Representative control-theoretic results on cyber-physical attacks

\begin{tabular}{ll}
\hline Attack name & References \\
\hline Replay attack & {$[5,47,48]$} \\
Dynamic false-data injection attack & {$[35,49]$} \\
$\begin{array}{l}\text { Stealth / false-data injection attack } \\
\text { (bias, surge, geometric) }\end{array}$ & {$[48,50-53]$} \\
Covert attack & {$[16,54]$}
\end{tabular}

control theory to model undetectable and unidentifiable attacks. Those attacks are equivalent to the aforementioned covert attack by Smith et al., where the adversary knows perfectly the system model dynamics. The parametric cyber-physical adversary defined in our work deals with equivalent scenarios.

Implementation of experimental testbeds Research on cyber-physical systems has progressed substantially resulting in a large number of experimental testbeds developed and established in the literature. Myat-Aung present in [37] a Secure Water Treatment (SWaT) simulation and testbed to test defense mechanisms against a variety of attacks. Siaterlis et al. [38] define a cyber-physical Experimentation Platform for Internet Contingencies (EPIC) that is able to study multiple independent infrastructures and to provide information about the propagation of faults and disruptions. Green et al. [39] focus their work on an adaptive cyber-physical testbed where they include different equipments, diverse networks, and also business processes. Yardley reports in [40] a cyberphysical testbed based on commercial tools in order to experimentally validate emerging research and technologies. The testbed combines emulation, simulation, and real hardware to experiment with smart grid technologies. Sanchez Arago et al. present in [41] a framework that is able to assess remotely the security of these systems. Krotofil and Larsen show in [42] several testbeds and simulations concluding that a successful attack against their envisioned systems has to manage cyber and physical knowledge.

From a more control-theoretic standpoint, Candell et al. report in [43] a testbed to analyze the performance of security mechanisms for cyber-physical systems. The work reports as well discussions from control and security practitioners. McLaughlin et al. analyze in [44] different testbeds and conclude that it is necessary to use pathways between cyber and physical components of the system in order to detect attacks. Also, Koutsandria et al. [45] implement a testbed where the data are crosschecked, using cyber and physical elements. Holm et al. survey, classify, and analyze in [46] several cyber-physical testbeds proposed for scientific research. Inline with the aforementioned contributions, we have presented in this paper a testbed that aims at evaluating mitigation techniques targeting attacks at the physical layer of cyberphysical systems operated via SCADA protocols. The goal of our testbed is to evaluate the control-theoretic security mechanism provided in this paper, under real-world constraints.

\section{Conclusions}

We have addressed security issues in cyber-physical systems. We have focused on the adaptation of failure 
detection mechanisms. The goal is to handle, in addition to faults and errors, the detection of attacks against industrial environments that close their loops through networked-control systems, more specifically cyber-physical attacks, i.e., attacks against cyber and physical layer of these systems.

We have reviewed the watermark-based detector proposed in $[4,5]$. We have shown that the detector fails at properly handling attacks carried out by cyber-physical adversaries. In particular, we have shown that an adversary that is able to acquire knowledge about the system model is able to succeed at attacking the system without being detected. Then, we have presented an enhanced detection scheme. The main idea of the new scheme is to use multiple watermark distributions and non-stationary identification signals. The resulting approach is able to detect both cyber and non-parametric cyber-physical adversaries. We have also shown that the new watermark-based detector works against a parametric cyber-physical adversary who knows only a set of control inputs. Nevertheless, if the adversary knows all the control inputs and sensor measurements of the system and uses the correct orders range with a window size sufficiently long, the watermark-based detector fails.

\section{Acknowledgements}

The authors acknowledge support from the Cyber CNI Chair of Institut Mines-Télécom (cf. http://chaire-cyber-cni.fr/). The chair is supported by Airbus Defence and Space, Amossys, EDF, Orange, La Poste, Nokia, Société Générale, and the Regional Council of Brittany. It has been acknowledged by the Center of Excellence in Cybersecurity.

\section{Authors' contributions}

All authors contributed equally to the manuscript. In addition, JRH carried out the numeric simulations and laboratory experiments and drafted the initial version of the manuscript. All authors read and approved the final manuscript.

\section{Competing interests}

The authors declare that they have no competing interests.

\section{Publisher's Note}

Springer Nature remains neutral with regard to jurisdictional claims in published maps and institutional affiliations.

\section{Author details}

1SAMOVAR, Telecom SudParis, CNRS, Université Paris-Saclay, 9 Rue Charles Fourier, 91000, Evry, France. ${ }^{2}$ Dipartimento di Ingegneria Elettrica e dell'Informazione, Politecnico di Bari, Via Orabona n.4, 70125, Bari, Italy.

Received: 13 February 2017 Accepted: 25 April 2017

Published online: 02 June 2017

\section{References}

1. D Corman, V Pillitteri, S Tousley, M Tehranipoor, U Lindqvist, NITRD Cyber-Physical Security Panel 35th IEEE Symposium on Security and Privacy. (IEEE S\&P, San Jose, CA, 2014)

2. N Falliere, LO Murchu, E Chien, W32. stuxnet dossier. White paper, Symantec Corp., Secur. Response. 5, 6 (2011)
3. J Rubio-Hernan, L De Cicco, J Garcia-Alfaro, in 11th International Conference on Availability, Reliability and Security. Revisiting a watermark-based detection scheme to handle cyber-physical attacks (IEEE, Salzburg, 2016)

4. Y Mo, B Sinopoli, in Communication, Control, and Computing. 47th Annual Allerton Conference On. Secure control against replay attacks (IEEE, 2009), pp. 911-918. doi:10.1109/ALLERTON.2009.5394956

5. Y Mo, S Weerakkody, B Sinopoli, Physical authentication of control systems: designing watermarked control inputs to detect counterfeit sensor outputs. IEEE Control Syst. 35(1), 93-109 (2015). doi:10.1109/MCS.2014.2364724

6. J Rubio-Hernan, L De Cicco, J Garcia-Alfaro, in Secure IT Systems: 21st Nordic Conference, NordSec 2016, Oulu, Finland, November 2-4, 2016. Proceedings. Event-triggered watermarking control to handle cyber-physical integrity attacks (Springer, Cham, 2016), pp. 3-19. doi:10.1007/978-3-319-47560-8_1

7. S Brown, Functional safety of electrical/electronic/programmable electronic safety related systems. Comput. Control Eng. J. 11(11), 14 (2000)

8. J Åkerberg, M Björkman, in Computer Safety, Reliability, and Security: 28th International Conference, SAFECOMP 2009, Hamburg, Germany, September 15-18, 2009. Proceedings. Exploring network security in PROFIsafe (Springer, Berlin, Heidelberg, 2009), pp. 67-80. doi:10.1007/978-3-642-04468-7_7

9. PROFIBUS and PROFINET International, International Standard, PROFINET Security Guideline (2013). http://www.profibus.com/download/ specifications-standards/, Accessed date October 2016

10. International Electrotechnical Commission, Industrial communication networks - Fieldbus specifications - Part 6-2: Application layer protocol specification - Type 2 elements (2014). https://webstore.iec.ch/ publication/4695, Accessed date Octobre 2016

11. J Rinaldi, ETHERNET/IP overview (2014). http://www.rtaautomation.com/ technologies/ethernetip/, Accessed date Octobre 2016

12. ED Knapp, Industrial Network Security: Securing Critical Infrastructure Networks for Smart Grid, SCADA, and Other Industrial Control Systems, 1st edn. (Syngress Publishing, Boston, 2011)

13. F Pasqualetti, Secure control systems: a control-theoretic approach to cyber-physical security. PhD thesis. (Department of Mechanical Engineering, University of California, Santa Barbara, 2012)

14. Q Zhu, Baş,ar, A hierarchical security architecture for smart grid. (Cambridge University Press, Cambridge, 2012), pp. 413-438. doi:10.1017/CBO9781139013468.019. Cambridge Books Online

15. A Teixeira, I Shames, H Sandberg, KH Johansson, A secure control framework for resource-limited adversaries. Automatica. 51, 135-148 (2015). doi:10.1016/j.automatica.2014.10.067

16. RS Smith, Covert misappropriation of networked control systems: presenting a feedback structure. IEEE Control Syst. 35(1), 82-92 (2015). doi:10.1109/MCS.2014.2364723

17. A Arvani, VS Rao, Detection and protection against intrusions on smart grid systems. Int. J. Cyber-Security Digital Forensics (IJCSDF). 3(1), 38-48 (2014)

18. VL Do, L Fillatre, I Nikiforov, in 2014 IEEE Conference on Control Applications (CCA). A statistical method for detecting cyber/physical attacks on SCADA systems, (2014), pp. 364-369. doi:10.1109/CCA.2014.6981373

19. GF Franklin, JD Powell, ML Workman, Digital Control of Dynamic Systems, 3rd edn. (Addison-Wesley Longman Publishing Co., Inc., Boston, 1998)

20. B Brumback, M Srinath, A chi-square test for fault-detection in Kalman filters. IEEE Trans. Automatic Control. 32(6), 552-554 (1987). doi:10.1109/TAC.1987.1104658

21. S Tripathi, MA Ikbal, Step size optimization of LMS algorithm using aunt colony optimization \& its comparison with particle swarm optimization algorithm in system identification. Int. Res. J. Eng. Technol. (IRJET). 2, 599-605 (2015)

22. H Natke, System identification: Torsten Söderström and Petre Stoica. Automatica. 28(5), 1069-1071 (1992)

23. B Widrow, JM McCool, MG Larimore, CR Johnson Jr, Stationary and nonstationary learning characteristics of the LMS adaptive filter. Proc. IEEE. 64(8), 1151-1162 (1976). doi:10.1109/PROC.1976.10286

24. NL Ricker, Model predictive control of a continuous, nonlinear, two-phase reactor. J. Process Control. 3(2), 109-123 (1993). doi:10.1016/0959-1524(93)80006-W

25. R Chabukswar, Y Mo, B Sinopoli, Detecting integrity attacks on SCADA systems. \{IFAC\} Proc. Volumes. 44(1), 11239-11244 (2011). doi:10.3182/20110828-6-IT-1002.03712 
26. L Ljung, Perspectives on system identification. Ann. Rev. Control. 34(1), 1-12 (2010). doi:10.1016/j.arcontrol.2009.12.001

27. M Barenthin Syberg, Complexity issues, validation and input design for control in system identification. PhD thesis (2008)

28. AA Cardenas, S Amin, S Sastry, in The 28th International Conference on Distributed Computing Systems Workshops. Secure control: towards survivable cyber-physical systems (IEEE, 2008), pp. 495-500. doi:10.1109/ICDCS.Workshops.2008.40

29. AA Cardenas, S Amin, B Sinopoli, A Giani, A Perrig, S Sastry, in Workshop on Future Directions in Cyber-Physical Systems Security. Challenges for securing cyber physical systems, (2009), p. 7. DHS. http://chess.eecs.berkeley.edu/ pubs/601.html

30. EK Wang, Y Ye, X Xu, S Yiu, L Hui, K Chow, in Proceedings of the 2010 IEEE/ACM Int'L Conference on Green Computing and Communications \& Int'L Conference on Cyber, Physical and Social Computing. GREENCOM-CPSCOM '10. Security issues and challenges for cyber physical system (IEEE Computer Society, Washington, DC, 2010), pp. 733-738. doi:10.1109/GreenCom-CPSCom.2010.36

31. C-W Ten, C-C Liu, G Manimaran, Vulnerability assessment of cybersecurity for scada systems. IEEE Trans. Power Syst. 23(4), 1836-1846 (2008). doi:10.1109/TPWRS.2008.2002298

32. B Zhu, A Joseph, S Sastry, in Internet of Things (iThings/CPSCom), 2011 International Conference on and 4th International Conference on Cyber, Physical and Social Computing. A Taxonomy of cyber attacks on SCADA systems (IEEE, 2011), pp. 380-388. doi:10.1109/iThings/CPSCom.2011.34

33. A Hahn, A Ashok, S Sridhar, M Govindarasu, Cyber-physical security testbeds: architecture, application, and evaluation for smart grid. IEEE Trans. Smart Grid. 4(2), 847-855 (2013). doi:10.1109/TSG.2012.2226919

34. M Mallouhi, Y Al-Nashif, D Cox, T Chadaga, S Hariri, in Innovative Smart Grid Technologies (ISGT), 2011 IEEE PES. A testbed for analyzing security of SCADA control systems (TASSCS), (2011), pp. 1-7. doi:10.1109/ISGT.2011.5759169

35. Y Mo, E Garone, A Casavola, B Sinopoli, in 49th IEEE Conference on Decision and Control (CDC). False data injection attacks against state estimation in wireless sensor networks, (2010), pp. 5967-5972. doi:10.1109/CDC.2010.5718158

36. F Pasqualetti, F Dorfler, F Bullo, in 2012 IEEE 51st IEEE Conference on Decision and Control (CDC). Cyber-physical security via geometric control: distributed monitoring and malicious attacks, (2012), pp. 3418-3425. doi:10.1109/CDC.2012.6426257

37. A Kaung Myat, Secure water treatment testbed (SWaT): an overview (2015). https://itrust.sutd.edu.sg/wp-content/uploads/sites/3/2015/11/ Brief-Introduction-to-SWaT_181115.pdf, Accessed date October 2016

38. C Siaterlis, B Genge, M Hohenadel, EPIC: a testbed for scientifically rigorous cyber-physical security experimentation. IEEE Trans. Emerging Topics Comput. 1(2), 319-330 (2013). doi:10.1109/TETC.2013.2287188

39. B Green, D Hutchison, SAF Frey, A Rashid, in Proceedings of the First International Workshop on Security and Resilience of Cyber-Physical Infrastructures (SERECIN). Testbed diversity as a fundamental principle for effective ICS security research (Lancaster University, Technical Report SCC-2016-01, 2016), pp. 12-15

40. TYardley, Testbed cross-cutting research (2014). https://tcipg.org/ research/testbed-cross-cutting-research, Accessed date October 2016

41. Aragó, ER Martínez, SS Clares, in Proceedings of the 2Nd International Symposium on ICS \& SCADA Cyber Security Research 2014. ICS-CSR 2014. SCADA Laboratory and test-bed as a service for critical infrastructure protection (BCS, UK, 2014), pp. 25-29. doi:10.14236/ewic/ics-csr2014.4, http://dx.doi.org/10.14236/ewic/ics-csr2014.4

42. M Krotofil, J Larsen, in DefCon 23. Rocking the pocket book: Hacking chemical plants for competition and extortion, vol. 23, (Las Vegas, 2015). https://www.blackhat.com/docs/us-15/materials/us-15-Krotofil-RockingThe-Pocket-Book-Hacking-Chemical-Plant-For-Competition-AndExtortion-wp.pdf

43. R Candell, K Stouffer, D Anand, in Process Control and Safety Symposium, International Society of Automation. A cybersecurity testbed for industrial control systems, (Houston, TX, 2014)

44. S McLaughlin, C Konstantinou, X Wang, L Davi, A-R Sadeghi, M Maniatakos, R Karri, The cybersecurity landscape in industrial control systems, vol. 104, (2016), pp. 1039-1057. doi:10.1109/JPROC.2015.2512235

45. G Koutsandria, R Gentz, M Jamei, A Scaglione, S Peisert, C McParland, in 1st ACM Workshop on Cyber-Physical Systems-Security And/or Privacy. A real-time testbed environment for cyber-physical security on the power grid (ACM, 2015), pp. 67-78

46. H Holm, M Karresand, A Vidström, E Westring, in Secure IT Systems: 20th Nordic Conference, NordSec 2015, Stockholm, Sweden, October 19-21, 2015, Proceedings, ed. by S Buchegger, M Dam. A Survey of industrial control system testbeds (Springer, Cham, 2015), pp. 11-26. doi:10.1007/978-3-319-26502-5_2

47. Y Mo, R Chabukswar, B Sinopoli, Detecting integrity attacks on SCADA systems. IEEE Trans. Control Syst. Technol. 22(4), 1396-1407 (2014). doi:10.1109/TCST.2013.2280899

48. A Teixeira, D Pérez, H Sandberg, KH Johansson, in Proceedings of the 1st International Conference on High Confidence Networked Systems. HiCoNS '12. Attack models and scenarios for networked control systems (ACM, New York, NY, 2012), pp. 55-64. doi:10.1145/2185505.2185515

49. F Pasqualetti, F Dorfler, F Bullo, Control-theoretic methods for cyberphysical security: geometric principles for optimal cross-layer resilient control systems. IEEE Control Syst. 35(1), 110-127 (2015). doi:10.1109/MCS.2014.2364725

50. G Dán, H Sandberg, in Smart Grid Communications (SmartGridComm), 2010 First IEEE International Conference On. Stealth attacks and protection schemes for state estimators in power systems, (2010), pp. 214-219. doi:10.1109/SMARTGRID.2010.5622046

51. Y Liu, P Ning, MK Reiter, False data injection attacks against state estimation in electric power grids. ACM Trans. Inform. Syst. Secur. (TISSEC). 14(1), 13 (2011)

52. RB Bobba, KMRQ Wang, H Khurana, K Nahtstedt, TJ Overbye, in Proceeding of the 1st Workshop on Secure Control Systems (CPSWEEK). Detecting false data injection attacks on DC state estimation (Citeseer, Stockholm, 2010), pp. 1-9

53. AA Cárdenas, S Amin, Z-S Lin, Y-L Huang, C-Y Huang, S Sastry, in Proceedings of the 6th ACM Symposium on Information, Computer and Communications Security. Attacks against process control systems: risk assessment, detection, and response. ASIACCS '11 (ACM, New York, NY, 2011), pp. 355-366. doi:10.1145/1966913.1966959

54. A decoupled feedback structure for covertly appropriating networked control systems. \{IFAC\} Proc. 44(1), 90-95 (2011). doi:10.3182/20110828-6-IT-1002.01721

\section{Submit your manuscript to a SpringerOpen ${ }^{\circ}$ journal and benefit from:}

- Convenient online submission

- Rigorous peer review

- Immediate publication on acceptance

- Open access: articles freely available online

- High visibility within the field

- Retaining the copyright to your article

Submit your next manuscript at $>$ springeropen.com 